\title{
Gauge- and renormalization-group-invariant formulation of the Higgs-boson resonance
}

\author{
Joannis Papavassiliou ${ }^{1}$ and Apostolos Pilaftsis ${ }^{2}$ \\ ${ }^{1}$ Theory Division, CERN, CH-1211 Geneva 23, Switzerland \\ ${ }^{2}$ Max-Planck-Institut für Physik, Föhringer Ring 6, 80805 Munich, Germany
}

(Received 16 October 1997; published 16 July 1998)

\begin{abstract}
A gauge- and renormalization-group-invariant approach implemented by the pinch technique is formulated for resonant transitions involving the Higgs boson. The line shape of the Higgs boson is shown to consist of two distinct and physically meaningful contributions: a process-independent resonant part and a processdependent non-resonant background, which are separately gauge independent, invariant under the renormalization group, satisfy naive, tree-level Ward identities, and respect the optical and equivalence theorem individually. The former process-independent quantity serves as the natural extension of the concept of the effective charge to the case of the Higgs scalar, and constitutes a common ingredient of every Born-improved amplitude. The difference in the phenomenological predictions obtained within our approach and those found with other methods is briefly discussed. [S0556-2821(98)07215-4]

PACS number(s): 14.80.Bn, 11.10.Gh, 11.15.Ex, 12.15.Lk
\end{abstract}

\section{INTRODUCTION}

The production of the Higgs boson, the only as yet unobserved building block of the standard model (SM), and the detailed study of its properties will be of central interest for several years to come. The Higgs boson is intimately connected to the prevailing field-theoretic mechanism for endowing gauge bosons, leptons, and quarks with masses [1]. Since understanding the origin of mass constitutes a major challenge for all models aspiring to describe physics beyond the SM, accurate experimental information about the Higgs sector is indispensable for determining both their theoretical relevance and their phenomenological viability.

Within the SM, the mass $M_{H}$ of the Higgs boson is a free parameter. The experimental lower bound on the SM Higgs boson through direct searches at the CERN Large Electron Positron collider (LEP) is $M_{H}>65.2 \mathrm{GeV}$ [2], whereas the theoretical upper bound is about $700 \mathrm{GeV}[3,4]$. Since the SM observables depend logarithmically on $M_{H}$ [5], the high precision electroweak data, even though they favor slightly a "light" Higgs boson of about $150 \mathrm{GeV}$, they can only impose rather loose bounds on $M_{H}$. In particular, from the LEP data on $\sin ^{2} \theta_{\text {eff }}^{\text {lept }}$, the electroweak observable most sensitive to $M_{H}$, the upper bound $M_{H}<550 \mathrm{GeV}$ is obtained at the $1.64 \sigma$ level [6], whereas a tighter upper bound of $M_{H}$ $<443 \mathrm{GeV}$ at the $1.64 \sigma$ has been advocated [7] after the inclusion of two-loop top quark effects in the calculations of $\sin ^{2} \theta_{\text {eff }}^{\text {lept }}[8]$.

A Higgs boson with mass of about $100 \mathrm{GeV}$ can be discovered at LEP2 [9], through the Bjorken process, or Higgsstrahlung, $e^{+} e^{-} \rightarrow Z H$ [10]. If the Higgs boson turns out to be heavier, its discovery will become again possible at the CERN Large Hadron Collider (LHC). In that case, the Higgs-boson production will proceed through a variety of sub-processes. In all of the above scenarios, depending on the value of $M_{H}$ and the specific kinematic conditions, the Higgs-boson production may be resonant. At that point, exactly as has happened in the case of the resonant Z-boson production [11], the well-known theoretical problems asso- ciated with the self-consistent treatment of resonant transition amplitudes are bound to resurface, but with the additional phenomenological complication that, in contrast to the $Z$-boson case, bosonic and fermionic channels give numerically comparable contributions to the Higgs-boson decay rate.

From the theoretical point of view, the self-consistent treatment of the Higgs boson resonance in the context of the SM has attracted significant attention, due to a variety of open questions [12]. In the vicinity of resonances transition amplitude become singular and must be regulated by a BreitWigner type of propagator. The most obvious signal that a method more sophisticated than a standard resummation of conventional self-energy graphs is needed in the case of nonAbelian gauge theories, comes from the simple calculational fact that the bosonic radiative corrections to the self-energies of vector $(\gamma, W, Z)$ or scalar (Higgs) bosons induce a nontrivial dependence on the gauge-fixing parameter (GFP), used to define the tree-level bosonic propagators appearing in the quantum loops. This is to be contrasted to the fermionic radiative corrections, which, even in the context of nonAbelian gauge theories behave as in quantum electrodynamics (QED), i.e., they are GFP independent. In addition, formal field-theoretic considerations as well as direct calculations show that, contrary to the QED case, the non-Abelian Green's functions do not satisfy their naive, tree-level Ward identities (WI's), after bosonic one-loop corrections are included. A careful analysis shows that this fundamental difference between Abelian and non-Abelian theories has farreaching consequences; the naive generalization of the BreitWigner method to the latter case gives rise to Born-improved amplitudes, which do not faithfully capture the underlying dynamics. Most noticeably, due to violation of the optical theorem (OT), unphysical thresholds and artificial resonances appear, which distort the Higgs boson lineshape. In addition, the high energy properties of such amplitudes are altered, and are in direct contradiction with the equivalence theorem (ET) $[13,14]$.

Recently however, a formalism based on the pinch technique (PT) [15-17] has been developed in a series of papers 
$[18,19]$ which bypasses all the aforementioned difficulties, and provides a self-consistent framework for dealing with unstable particles and resonant transition amplitudes in the context of non-Abelian gauge theories. In [18] the general methodology has been presented, whereas in [19] the crucial physical requirements for a physically meaningful resummation have been discussed in detail. In addition, it was shown that the resummation algorithm based on the PT satisfies all those requirements; in fact, to the best of our knowledge, it is the only algorithm known to date which can accomplish this. Several applications of the above formalism may be found in the literature [20]. In this paper we employ the above formalism in order to develop a systematic approach to resonant transition amplitudes involving the SM Higgs boson. The theoretical highlights of our study have been presented in a short communication [21]. In this longer paper we address in detail the most important calculational aspects of this analysis, and discuss extensively the multitude of physical issues involved.

The paper is organized as follows: In Sec. II, we use the PT to compute the GFP-independent Higgs-boson selfenergy at the one-loop level, within the context of three different characteristic gauges, namely the Feynman-t'Hooft gauge, the general renormalizable $R_{\xi}$ gauges, and the covariant background field gauges (BFG's). Explicit expressions are reported, and the pathologies associated with gauge dependences in the conventional formulation are discussed. In Sec. III, we employ arguments of unitarity and analyticity and show how the effective Higgs-boson self-energy of the previous section may be obtained from tree-level amplitudes involving the Higgs boson; in fact, it satisfies individually the OT, both for fermionic as well as bosonic contributions. In addition, we apply our formalism to the process $t \bar{t} \rightarrow H^{*}$ $\rightarrow t \bar{t}$, and discuss how our predictions differ from those obtained by other methods. In Sec. IV, we review the notion of the effective charge in QED and discuss how this concept may be extended to the case of gluon in quantum chromodynamics (QCD), based on properties of the PT gluon selfenergy under the renormalization group [15]. Furthermore, we demonstrate explicitly that in pure scalar theories, e.g., $\left(\phi^{3}\right)_{6}$ in six space-time dimensions, the scalar particle does not admit the construction of a renormalization-groupinvariant (RGI) quantity which could serve as the analog of the QED effective charge. However, if the scalar theory is endowed with a global symmetry, which, in turn, is broken spontaneously, we find that a scalar effective "charge" may still be formed. This latter example provides useful insight and sets up the stage for addressing the more complicated case of the full SM. The next two sections contain the main theoretical thrust of our paper: In Sec. V, after discussing how the process-independent PT self-energies for the $W$ and $Z$ bosons of the SM can give rise to RGI quantities which may be identified as effective charges [22], we show that the construction of a process-independent and RGI quantity involving the Higgs-boson propagator is indeed possible. The above construction of a Higgs-boson effective charge becomes only possible by virtue of the naive, tree-level WI's satisfied by the GFP-independent PT sub-amplitudes. In Sec.

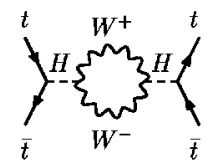

(a)

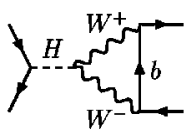

(e)

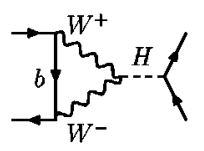

(i)

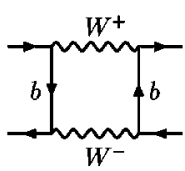

(m)

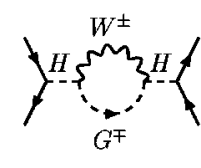

(b)

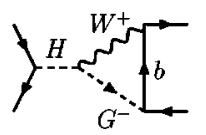

(f)

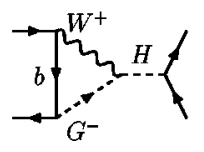

(j)

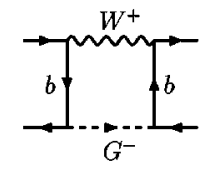

(n)

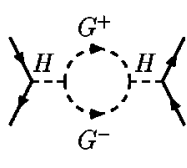

(c)

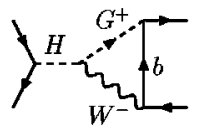

(g)

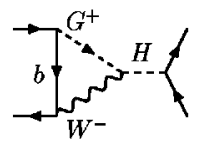

(k)

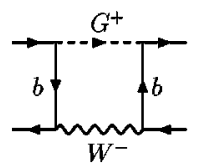

(o)

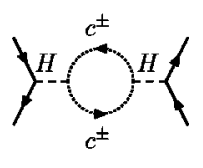

(d)

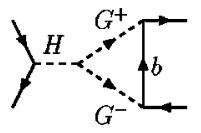

(h)

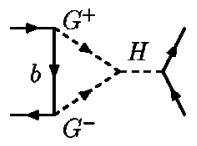

(1)

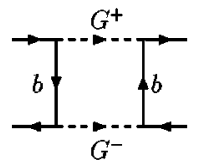

(p)
FIG. 1. The Higgs-mediated part of the one-loop amplitude $t \bar{t} \rightarrow t \bar{t}$.

VI, we show with an explicit example that the PT subamplitudes satisfy the ET individually, and that with the help of the same PT WI's, this fact remains true even after resummation. In Sec. VII, we present our conclusions. Finally, lengthy analytic expressions pertaining to the $H W W$ and $H Z Z$ vertices are relegated to the Appendices.

\section{ONE LOOP CALCULATIONS IN THE PINCH TECHNIQUE}

In this section we show how the application of the PT gives rise to an effective self-energy for the Higgs boson, which is independent of the GFP, and displays a high-energy behavior which is consistent with the ET. For definiteness we focus on the gauge invariant subset of Feynman diagrams containing two $W$ bosons (and their corresponding would-be Goldstone bosons and ghosts) in a typical S-matrix element, e.g., $t \bar{t} \rightarrow H^{*} \rightarrow t \bar{t}$, shown in Fig. 1. We carry out this calculation in three representative gauges, i.e., the renormalizable Feynman-'t Hooft gauge, the general $R_{\xi}$ gauges, and the background field method (BFM) in the covariant $R_{\xi_{Q}}$ gauges. We discuss the relevant technical points and present explicit intermediate and final results.

\section{A. The Feynman-'t Hooft gauge}

First we present the calculation for the special GFP choice $\xi=1$ in the renormalizable $R_{\xi}$ gauges. This particular choice is known to simplify computations; of course, as we will see explicitly in the next subsection, the same final answer emerges for any other choice of $\xi$, after the PT algorithm has been carried out.

We first calculate the diagrams contributing to the conventional Higgs boson self-energy [Figs. 1(a)-1(d)]. A 
straightforward calculation yields (we omit contributions from tadpole and seagull graphs):

$$
\Pi^{H H}\left(q^{2}\right)=\frac{\alpha_{w}}{4 \pi}\left(-q^{2}+3 M_{W}^{2}+\frac{M_{H}^{4}}{4 M_{W}^{2}}\right) B_{0}\left(q^{2}, M_{W}^{2}, M_{W}^{2}\right),
$$

where $\alpha_{w}=g_{w}^{2} /(4 \pi)$ and

$$
\begin{aligned}
& B_{0}\left(p^{2}, m_{1}^{2}, m_{2}^{2}\right) \\
& \quad=(2 \pi \mu)^{4-d} \int \frac{d^{d} k}{i \pi^{2}} \frac{1}{\left(k^{2}-m_{1}^{2}\right)\left[(k+p)^{2}-m_{2}^{2}\right]}
\end{aligned}
$$

is the Veltman-'t Hooft function [23] defined in $d=4-2 \epsilon$ dimensions, using the conventions of Ref. [24]. From the integrand of Eq. (2.2), it is clear that $B_{0}\left(p^{2}, m_{1}^{2}, m_{2}^{2}\right)$ develops absorptive (imaginary) parts, when $\sqrt{p^{2}} \geqslant m_{1}+m_{2}$. The mass parameters $m_{1}, m_{2}$ may represent either physical masses, such as that of the $W$ and/or $Z$ bosons, or masses of the respective unphysical would-be Goldstone bosons and ghosts.

According to the PT [15], we must now extract the propagator-like pieces concealed inside vertex and box diagrams. Such pieces emerge every time longitudinal momenta coming from propagators or vertices trigger elementary WI's of the form

$$
k P_{L}=\left(k+\not p-m_{b}\right) P_{L}-P_{R}\left(\not p-m_{t}\right)+m_{b} P_{L}-m_{t} P_{R},
$$

where $P_{R(L)}=\left[1+(-) \gamma_{5}\right] / 2$ is the chirality projection operator. The first term in Eq. (2.3) pinches out the internal propagator of the $b$ quark, whereas the second one dies when contracted with the spinor of the external on-shell $t$ quark.

In the Feynman-'t Hooft gauge, the only graphs that can give rise to propagator-like contributions are the vertex graphs of Figs. 1(f) and 1(g), denoted by $\mathcal{V}_{G W}$ and $\mathcal{V}_{W G}$, and their mirror images, Figs. 1(j) and 1(k). Setting $p^{\prime}=p-q$, we define $S_{R}^{\nu}$ and $S_{L}^{\nu}$ as follows:

$$
\begin{aligned}
& S_{R}^{\nu}=\frac{g_{w}^{2}}{2} \bar{v}\left(p^{\prime}\right)\left(m_{t} P_{L}-m_{b} P_{R}\right) \frac{1}{k+p-m_{b}} \gamma^{\nu} P_{L} u(p), \\
& S_{L}^{\mu}=\frac{g_{w}^{2}}{2} \bar{v}\left(p^{\prime}\right) \gamma^{\mu} P_{L} \frac{1}{k+p-m_{b}}\left(m_{t} P_{R}-m_{b} P_{L}\right) u(p) .
\end{aligned}
$$

The action of the longitudinal momenta from the vertices on these expressions gives

$$
\begin{array}{r}
k_{\nu} S_{R}^{\nu}=\frac{g_{w}^{2}}{2} \bar{v}\left(p^{\prime}\right) m_{t} P_{L} u(p)+\cdots, \\
(k+q)_{\mu} S_{L}^{\mu}=\frac{g_{w}^{2}}{2} \bar{v}\left(p^{\prime}\right) m_{t} P_{R} u(p)+\cdots,
\end{array}
$$

where the ellipses mean omission of vertex like pieces, e.g., pieces still containing the $b$ quark tree-level propagator, $(k$ $\left.+\not p-m_{b}\right)^{-1}$. With the help of Eq. (2.6), we find

$$
\begin{aligned}
\mathcal{V}_{W G}+\mathcal{V}_{G W}= & -\left[(2 k+q)_{\nu} S_{R}^{\nu}+(2 k+q)_{\mu} S_{L}^{\mu}\right] \\
& \times B_{0}\left(q^{2}, M_{W}^{2}, M_{W}^{2}\right) \\
= & -\frac{\alpha_{w}}{8 \pi} m_{t} B_{0}\left(q^{2}, M_{W}^{2}, M_{W}^{2}\right) \bar{v}\left(p^{\prime}\right) u(p)+\cdots .
\end{aligned}
$$

We notice that the only propagator-like piece couples to the external $t \bar{t}$ pair exactly as a Higgs boson. In addition, no term proportional to $\gamma_{5}$ has survived; had such a term been present, it ought to be alloted to the effective $H G^{0}$ one-loop mixing self-energy, thus breaking the $\mathrm{CP}$ invariance of the underlying theory [25]. This exercise demonstrates explicitly how the PT preserves the discrete symmetries of the classical action after quantization.

Finally, the pinch contribution $\mathcal{V}_{G W}$ to the Higgs-boson self-energy stemming from the vertex (mirror image graphs give an extra factor of 2) is

$$
\mathcal{V}_{G W}=-\frac{\alpha_{w}}{4 \pi}\left(q^{2}-M_{H}^{2}\right) B_{0}\left(q^{2}, M_{W}^{2}, M_{W}^{2}\right) .
$$

Adding the contribution from Eq. (2.8) to the conventional result of Eq. (2.1), we finally arrive at the following expression for the PT one-loop Higgs boson self-energy $\hat{\Pi}^{H H}\left(q^{2}\right)$,

$$
\begin{aligned}
\hat{\Pi}_{(W W)}^{H H}\left(q^{2}\right) & \\
= & \frac{\alpha_{w}}{16 \pi} \frac{M_{H}^{4}}{M_{W}^{2}}\left[1+4 \frac{M_{W}^{2}}{M_{H}^{2}}-4 \frac{M_{W}^{2}}{M_{H}^{4}}\left(2 q^{2}-3 M_{W}^{2}\right)\right] \\
& \times B_{0}\left(q^{2}, M_{W}^{2}, M_{W}^{2}\right) .
\end{aligned}
$$

\section{B. $R_{\xi}$ renormalizable gauges}

After this introductory calculation, we turn to the general case, where the GFP $\xi$ is kept arbitrary. In these gauges, the free $W$-boson propagator is given by

$$
\begin{aligned}
\Delta_{\mu \nu}^{\left(\xi_{w}\right)}(q) & =\left[-g_{\mu \nu}+\left(1-\xi_{w}\right) \frac{q_{\mu} q_{\nu}}{q^{2}-\xi_{w} M_{W}^{2}}\right] \frac{1}{q^{2}-M_{W}^{2}} \\
& =U_{\mu \nu}(q)-\frac{q_{\mu} q_{\nu}}{M_{W}^{2}} D^{\left(\xi_{w}\right)}\left(q^{2}\right),
\end{aligned}
$$

where

$$
D^{\left(\xi_{w}\right)}\left(q^{2}\right)=\frac{1}{q^{2}-\xi_{w} M_{W}^{2}}
$$

is the propagator of the would-be Goldstone boson and ghost fields. A straightforward calculation for the conventional one-loop Higgs self-energy yields

$$
\begin{aligned}
\Pi^{H H}\left(q^{2}\right)= & \frac{\alpha_{w}}{4 \pi}\left[\left(\frac{\left(q^{2}\right)^{2}}{4 M_{W}^{2}}-q^{2}+3 M_{W}^{2}\right) B_{0}\left(q^{2}, M_{W}^{2}, M_{W}^{2}\right)\right. \\
& \left.+\frac{M_{H}^{4}-\left(q^{2}\right)^{2}}{4 M_{W}^{2}} B_{0}\left(q^{2}, \xi_{w} M_{W}^{2}, \xi_{w} M_{W}^{2}\right)\right],
\end{aligned}
$$


where tadpole and seagull terms have again been omitted. Some comments are now in order regarding Eq. (2.12):

(a) The term proportional to $\left(q^{2}\right)^{2}$ is absent only for the special choice $\xi_{w}=1$, in which case $B_{0}$ factorizes out.

(b) The term proportional to $\left(q^{2}\right)^{2}$ is ultraviolet (UV) finite, i.e., it does not depend on the UV regulator $1 / \epsilon$ for any value of $\xi_{w}$. Of course, this is expected, since we are working within a renormalizable gauge.

(c) Even though terms proportional to $B_{0}\left(q^{2}, M_{W}^{2}, \xi_{w} M_{W}^{2}\right)$ appear in intermediate calculations of individual diagrams, they finally cancel in the sum. So, there are no terms with mixed poles; we only have thresholds at $q^{2}=4 M_{W}^{2}$ and $q^{2}=4 \xi_{w} M_{W}^{2}$. This result can be traced back to the fact that the tree-level $H W_{\mu}^{+} W_{\nu}^{-}$coupling is proportional to $g_{\mu \nu}$ and hence, any contraction between the longitudinal and transverse parts of the two $W$ boson propagators in the loop will vanish. The transverse part of the $W$-boson propagator is associated with the physical pole at $q^{2}=M_{W}^{2}$, whereas the longitudinal one possesses an unphysical singularity at $q^{2}=\xi M_{W}^{2}$. Since only terms arising from the contraction between transverse-transverse and longitudinal-longitudinal parts of the $W$-boson propagators can survive, the absence of mixed poles is expected. However, this last feature may change in higher orders, since new momentum-dependent form-factors for the vertex $H W_{\mu}^{+} W_{\nu}^{-}$are radiatively induced, which could give rise to mixed poles.

(d) Setting $\xi_{w}=1$ in the expression of Eq. (2.12), we recover the result of Eq. (2.1).

Next we collect the pinch contributions which are kinematically akin to a Higgs boson self-energy. Due to the additional longitudinal momenta proportional to $1-\xi_{w}$, we receive extra pinch contributions, from the vertex- as well as the box-diagrams. The only technically subtle point in this context is that the propagator-like parts related to the Higgs boson arise from two successive contractions of the longitudinal momenta on the elementary vertex: the first momentum pinches, giving rise to propagator-like terms whose coupling to the external quarks is proportional to $\gamma_{\mu} P_{L}$; clearly this coupling is not Higgs-boson-like. In addition, a vertex-like term proportional to $m_{t}$ survives. After the second longitudinal momentum is contracted with that latter vertex-like term, it removes the internal fermion propagator and gives rise to a propagator-like contribution, which couples to the external fermions proportionally to $m_{t}$. To see that mechanism in detail, consider the typical quantity $\mathcal{T}_{t}^{\mu \nu}$, appearing in the graphs in question, defined as

$$
\mathcal{T}_{t}^{\mu \nu}=\frac{g_{w}^{2}}{2} \bar{v}\left(p^{\prime}\right) \gamma^{\mu} P_{L} \frac{1}{k+\not p-m_{b}} \gamma^{\nu} P_{L} u(p) .
$$

The action of the first longitudinal momentum $k_{\nu}$ gives

$$
k_{\nu} V^{\mu \nu}=\frac{g_{w}^{2}}{2} \bar{v}\left(p^{\prime}\right) \gamma^{\mu} P_{L} u(p)+S_{L}^{\mu} .
$$

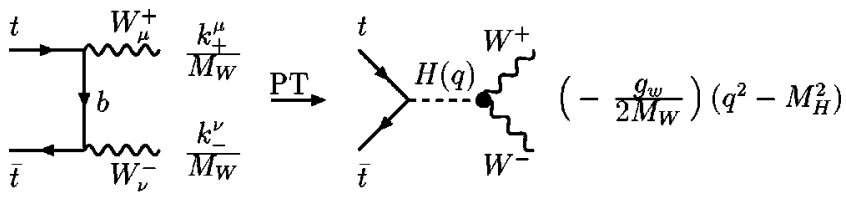

(a)

(b)

FIG. 2. Higgs-like contribution from the $t$-channel graph.

When the second momentum $k_{\mu}$ acts on the first term on the right-hand side (RHS) of Eq. (2.14), by virtue of Eq. (2.6) gives rise to a propagator-like term proportional to $m_{t}$, as is also shown in Fig. 2, according to Eq. (2.6). As for the second term on the RHS of Eq. (2.14), after it gets contracted with the second momentum $k_{\mu}$, it will be judiciously alloted to the various remaining effective self-energies, such as $\gamma \gamma, Z Z, \gamma Z, Z \gamma$, etc., according to the rules established in [26]. These latter terms are not displayed in Fig. 2.

We now gather all relevant pinch contributions from the box diagrams:

$$
\begin{aligned}
\mathcal{B}_{W W}\left(q^{2}\right)= & \frac{\alpha_{w}}{16 \pi M_{W}^{2}}\left[B_{0}\left(q^{2}, M_{W}^{2}, M_{W}^{2}\right)\right. \\
& -2 B_{0}\left(q^{2}, M_{W}^{2}, \xi_{w} M_{W}^{2}\right) \\
& \left.+B_{0}\left(q^{2}, \xi_{w} M_{W}^{2}, \xi_{w} M_{W}^{2}\right)\right]\left(q^{2}-M_{H}^{2}\right)^{2}, \\
\mathcal{B}_{G W}\left(q^{2}\right)= & \frac{\alpha_{w}}{8 \pi M_{W}^{2}}\left[B_{0}\left(q^{2}, M_{W}^{2}, \xi_{w} M_{W}^{2}\right)\right. \\
& \left.-B_{0}\left(q^{2}, \xi_{w} M_{W}^{2}, \xi_{w} M_{W}^{2}\right)\right]\left(q^{2}-M_{H}^{2}\right)^{2} .
\end{aligned}
$$

The net pinch contribution to the effective Higgs boson selfenergy originating from the box graphs may be summarized by

$$
\begin{aligned}
\mathcal{B}\left(q^{2}\right)= & \frac{\alpha_{w}}{16 \pi M_{W}^{2}}\left[B_{0}\left(q^{2}, M_{W}^{2}, M_{W}^{2}\right)\right. \\
& \left.-B_{0}\left(q^{2}, \xi_{w} M_{W}^{2}, \xi_{w} M_{W}^{2}\right)\right]\left(q^{2}-M_{H}^{2}\right)^{2} .
\end{aligned}
$$

Again, the terms proportional to $B_{0}\left(q^{2}, M_{W}^{2}, \xi_{w} M_{W}^{2}\right)$ cancel. In addition, for $\xi_{w}=1$, the above expression vanishes as it should, since in the Feynman gauge there are no pinch contributions coming from boxes.

Similarly, the individual pinch contributions from vertex graphs are listed below:

$$
\begin{aligned}
\mathcal{V}_{W W}\left(q^{2}\right)= & -\frac{\alpha_{w}}{4 \pi}\left[\left(1+\frac{q^{2}}{2 M_{W}^{2}}\right) B_{0}\left(q^{2}, M_{W}^{2}, M_{W}^{2}\right)\right. \\
& -\left(1-\xi_{w}+\frac{q^{2}}{M_{W}^{2}}\right) B_{0}\left(q^{2}, M_{W}^{2}, \xi_{w} M_{W}^{2}\right) \\
& \left.+\left(\frac{q^{2}}{2 M_{W}^{2}}-\xi_{w}\right) B_{0}\left(q^{2}, \xi_{w} M_{W}^{2}, \xi_{w} M_{W}^{2}\right)\right] \\
& \times\left(q^{2}-M_{H}^{2}\right),
\end{aligned}
$$




$$
\begin{aligned}
\mathcal{V}_{G W}\left(q^{2}\right)= & -\frac{\alpha_{w}}{4 \pi}\left[\left(1-\xi_{w}+\frac{q^{2}}{M_{W}^{2}}\right) B_{0}\left(q^{2}, M_{W}^{2}, \xi_{w} M_{W}^{2}\right)\right. \\
& \left.+\left(\xi_{w}-\frac{q^{2}}{M_{W}^{2}}\right) B_{0}\left(q^{2}, \xi_{w} M_{W}^{2}, \xi_{w} M_{W}^{2}\right)\right] \\
& \times\left(q^{2}-M_{H}^{2}\right)
\end{aligned}
$$

which gives in the sum

$$
\begin{aligned}
\mathcal{V}\left(q^{2}\right)= & -\frac{\alpha_{w}}{4 \pi}\left[\left(1+\frac{q^{2}}{2 M_{W}^{2}}\right) B_{0}\left(q^{2}, M_{W}^{2}, M_{W}^{2}\right)\right. \\
& \left.-\frac{q^{2}}{2 M_{W}^{2}} B_{0}\left(q^{2}, \xi_{w} M_{W}^{2}, \xi_{w} M_{W}^{2}\right)\right]\left(q^{2}-M_{H}^{2}\right) .
\end{aligned}
$$

Again, the terms proportional to $B_{0}\left(q^{2}, M_{W}^{2}, \xi_{w} M_{W}^{2}\right)$ cancel in the final result, and the analytic expression of Eq. (2.8) emerges for $\xi_{w}=1$.

Adding Eq. (2.17) and Eq. (2.20), we find that in the linear renormalizable gauges the total pinch contribution to effective Higgs boson self-energy is given by

$$
\begin{aligned}
\Pi^{H H, P}\left(q^{2}\right)= & -\frac{\alpha_{w}}{4 \pi}\left[\left(1+\frac{q^{2}+M_{H}^{2}}{4 M_{W}^{2}}\right) B_{0}\left(q^{2}, M_{W}^{2}, M_{W}^{2}\right)\right. \\
& \left.-\frac{q^{2}+M_{H}^{2}}{4 M_{W}^{2}} B_{0}\left(q^{2}, \xi_{w} M_{W}^{2}, \xi_{w} M_{W}^{2}\right)\right] \\
& \times\left(q^{2}-M_{H}^{2}\right) .
\end{aligned}
$$

Adding Eq. (2.21) to the conventional result given in Eq. (2.12), we see that all terms proportional to $B_{0}\left(q^{2}, \xi_{w} M_{W}^{2}, \xi_{w} M_{W}^{2}\right)$, which are the only terms depending on $\xi_{w}$, cancel, and we find again the PT result given in Eq. (2.9).

\section{The covariant background field gauge}

We shall consider the BFM applied to the covariant gauges [27-29]; a detailed discussion of the BFM in the non-covariant gauges may be found in [30]. The calculation here is particularly illuminating, because it shows that the results are plagued with pathologies away from $\xi_{Q}=1$ [19].

Using the Feynman rules of the covariant background field gauge [28], we obtain for the Higgs-boson self-energy in an arbitrary $\xi_{Q}$ gauge

$$
\begin{aligned}
\Pi^{\hat{H} \hat{H}}\left(q^{2}\right)= & \frac{\alpha_{w}}{4 \pi}\left\{\left(\frac{\left(q^{2}\right)^{2}}{4 M_{W}^{2}}-q^{2}+3 M_{W}^{2}\right) B_{0}\left(q^{2}, M_{W}^{2}, M_{W}^{2}\right)\right. \\
& +\left[\frac{M_{H}^{4}-\left(q^{2}\right)^{2}}{4 M_{W}^{2}}-\xi_{Q}\left(q^{2}-M_{H}^{2}\right)\right] \\
& \left.\times B_{0}\left(q^{2}, \xi_{Q} M_{W}^{2}, \xi_{Q} M_{W}^{2}\right)\right\} .
\end{aligned}
$$

Some important comments must be made:

(a) Setting $\xi_{Q}=1$ in the expression of Eq. (2.22), we recover the full PT answer of Eq. (2.9), in accordance with earlier observations [28,29]. (b) We see that for $\xi_{Q} \neq 1$ the $\left(q^{2}\right)^{2}$ term survives and is proportional to the difference $B_{0}\left(q^{2}, M_{W}^{2}, M_{W}^{2}\right)$ $-B_{0}\left(q^{2}, \xi_{Q} M_{W}^{2}, \xi_{Q} M_{W}^{2}\right)$. For any finite value of $\xi_{O}$ this term vanishes for sufficiently large $q^{2}$, i.e., $q^{2}$ $\gg M_{W}^{2}$ and $q^{2} \gg \xi_{Q} M_{W}^{2} \cdot{ }^{1}$ Therefore, the quantity in Eq. (2.22) displays good high energy behavior in compliance with unitarity. Notice however that the onset of this good behavior depends crucially on the choice of $\xi_{Q}$. Since $\xi_{Q}$ is a free parameter and may be chosen to be arbitrarily large, but finite, the restoration of unitarity may be arbitrarily delayed as well. This fact poses no problem as long as one is restricted to the computation of physical amplitudes at a finite order in perturbation theory. However, if the above self-energy was to be resummed in order to regulate resonant transition amplitudes, it would lead to an artificial delay of unitarity restoration. Specific quantitative examples of such artifacts will be presented in Sec. VII.

(c) In addition to the problem described above, which becomes significant for large values of $\xi_{Q}$, a serious pathology occurs for any value of $\xi_{Q} \neq 1$, namely the appearance of unphysical thresholds $[18,19]$. Such thresholds may be particularly misleading if $\xi_{Q}$ is chosen in the vicinity of unity, giving rise to distortions in the lineshape of the unstable particle.

We then proceed to isolate the propagator-like pinch parts from the BFG boxes and vertices, for general $\xi_{Q}$. Clearly, the box contributions are the same as in the linear renormalizable gauges; they can be recovered from Eq. (2.17) by the simple replacement $\xi_{w} \rightarrow \xi_{Q}$. The same is true for the pinch contributions involving the $W W$ virtual states, i.e., $\mathcal{V}_{W W}$ in Eq. (2.18). In this way, the total pinch box contribution, $\mathcal{B}$, and $\mathcal{V}_{W W}$ may be separately written down

$$
\begin{aligned}
\mathcal{B}\left(q^{2}\right)= & \frac{\alpha_{w}}{16 \pi M_{W}^{2}}\left[B_{0}\left(q^{2}, M_{W}^{2}, M_{W}^{2}\right)\right. \\
& \left.-B_{0}\left(q^{2}, \xi_{Q} M_{W}^{2}, \xi_{Q} M_{W}^{2}\right)\right] \\
& \times\left(q^{2}-M_{H}^{2}\right)^{2}, \\
\mathcal{V}_{W W}\left(q^{2}\right)= & -\frac{\alpha_{w}}{4 \pi}\left[\left(1+\frac{q^{2}}{2 M_{W}^{2}}\right) B_{0}\left(q^{2}, M_{W}^{2}, M_{W}^{2}\right)\right. \\
& -\left(1-\xi_{Q}+\frac{q^{2}}{M_{W}^{2}}\right) B_{0}\left(q^{2}, M_{W}^{2}, \xi_{Q} M_{W}^{2}\right) \\
& \left.+\left(\frac{q^{2}}{2 M_{W}^{2}}-\xi_{Q}\right) B_{0}\left(q^{2}, \xi_{Q} M_{W}^{2}, \xi_{Q} M_{W}^{2}\right)\right] \\
& \times\left(q^{2}-M_{H}^{2}\right) .
\end{aligned}
$$

However, the vertex graph $\mathcal{V}_{G W}$ is different, since the coupling between $\hat{H} G^{ \pm} W^{\mp}$ in the BFG differs from the respec-

\footnotetext{
${ }^{1} \mathrm{~A}$ noticeable exception is the unitary gauge $\left(\xi_{Q} \rightarrow \infty\right)$, in which such a term survives and, in fact, gives rise to a divergent, nonrenormalizable contribution.
} 
tive $H G^{ \pm} W^{\mp}$ coupling in the $R_{\xi}$ gauges $\left[2 q_{\mu}\right.$ as opposed to $(2 k+q)_{\mu}$, respectively]. Specifically, we have

$$
\begin{aligned}
\mathcal{V}_{G W}\left(q^{2}\right)= & -\frac{\alpha_{w}}{4 \pi}\left[\left(1-\xi_{Q}+\frac{q^{2}}{M_{W}^{2}}\right) B_{0}\left(q^{2}, M_{W}^{2}, \xi_{Q} M_{W}^{2}\right)\right. \\
& \left.-\frac{q^{2}}{M_{W}^{2}} B_{0}\left(q^{2}, \xi_{Q} M_{W}^{2}, \xi_{Q} M_{W}^{2}\right)\right]\left(q^{2}-M_{H}^{2}\right) .
\end{aligned}
$$

Adding both pinch terms in Eqs. (2.24) and (2.25), we easily obtain

$$
\begin{aligned}
\mathcal{V}\left(q^{2}\right)= & -\frac{\alpha_{w}}{4 \pi}\left[\left(1+\frac{q^{2}}{2 M_{W}^{2}}\right) B_{0}\left(q^{2}, M_{W}^{2}, M_{W}^{2}\right)\right. \\
& \left.-\left(\frac{q^{2}}{2 M_{W}^{2}}+\xi_{Q}\right) B_{0}\left(q^{2}, \xi_{Q} M_{W}^{2}, \xi_{Q} M_{W}^{2}\right)\right]\left(q^{2}-M_{H}^{2}\right) .
\end{aligned}
$$

Finally, the total pinch contribution to the Higgs boson selfenergy, which is obtained by forming the sum of Eqs. (2.23) and (2.26), is given by

$$
\begin{aligned}
\Pi^{\hat{H} \hat{H}, P}\left(q^{2}\right)= & -\frac{\alpha_{w}}{4 \pi}\left[\left(1+\frac{q^{2}}{4 M_{W}^{2}}+\frac{M_{H}^{2}}{4 M_{W}^{2}}\right) B_{0}\left(q^{2}, M_{W}^{2}, M_{W}^{2}\right)\right. \\
& -\left(\frac{q^{2}}{4 M_{W}^{2}}+\frac{M_{H}^{2}}{4 M_{W}^{2}}+\xi_{Q}\right) \\
& \left.\times B_{0}\left(q^{2}, \xi_{Q} M_{W}^{2}, \xi_{Q} M_{W}^{2}\right)\right]\left(q^{2}-M_{H}^{2}\right) .
\end{aligned}
$$

Adding Eq. (2.27) to Eq. (2.22), we arrive again at the expression of Eq. (2.9).

In a similar way, we may compute the contributions of the other virtual channels $(t \bar{t}, Z Z$, and $H H)$ to the effective Higgs boson self-energy. They are given by

$$
\begin{aligned}
\hat{\Pi}_{(t t)}^{H H}\left(q^{2}\right)= & \frac{3 \alpha_{w}}{8 \pi} \frac{m_{t}^{2}}{M_{W}^{2}}\left(q^{2}-4 m_{t}^{2}\right) B_{0}\left(q^{2}, m_{t}^{2}, m_{t}^{2}\right), \\
\hat{\Pi}_{(Z Z)}^{H H}\left(q^{2}\right)= & \frac{\alpha_{w}}{32 \pi} \frac{M_{H}^{4}}{M_{W}^{2}}\left[1+4 \frac{M_{Z}^{2}}{M_{H}^{2}}-4 \frac{M_{Z}^{2}}{M_{H}^{4}}\left(2 q^{2}-3 M_{Z}^{2}\right)\right] \\
& \times B_{0}\left(q^{2}, M_{Z}^{2}, M_{Z}^{2}\right), \\
\hat{\Pi}_{(H H)}^{H H}\left(q^{2}\right)= & \frac{9 \alpha_{w}}{32 \pi} \frac{M_{H}^{4}}{M_{W}^{2}} B_{0}\left(q^{2}, M_{H}^{2}, M_{H}^{2}\right) .
\end{aligned}
$$

Note that $\hat{\Pi}_{(t t)}\left(q^{2}\right)$ and $\hat{\Pi}_{(H H)}\left(q^{2}\right)$ are identical to their conventionally defined counterparts, i.e., they receive no pinch contributions.

\section{THE RESONANT HIGGS BOSON AND UNITARITY}

In this section we show how one can obtain the results of the previous section by resorting to the fundamental properties of unitarity and analyticity of $S$-matrix elements.

As explained in detail in [19], a close connection exists between gauge invariance and unitarity, which is best established by looking at the two sides of the equation for the OT. The OT for a given process $\langle b|T| a\rangle$ is

$$
\left\langle b\left|\left(T-T^{\dagger}\right)\right| a\right\rangle=i \sum_{m}(2 \pi)^{4} \delta^{4}\left(P_{m}-P_{a}\right)\langle m|T| b\rangle^{*}\langle m|T| a\rangle,
$$

where the sum $\Sigma_{m}$ should be understood to be over the entire phase space and spins of all possible on-shell intermediate particles $m$. The RHS of Eq. (3.1) consists of the product of GFP-independent on shell amplitudes, thus enforcing the gauge-invariance of the imaginary part of the amplitude on the LHS. In particular, even though the LHS contains unphysical particles, such as ghosts and would-be Goldstone bosons, which could give rise to unphysical thresholds, Eq. (3.1) guarantees that all such contributions will vanish. In general, the aforementioned cancellation takes place after contributions from the propagator-, vertex-, and boxdiagrams have been combined. There are field theories however, such as scalar theories, or QED, which allow for a stronger version of the equality given in Eq. (3.1): The optical relationship holds individually for the propagator-, vertex-, and box-diagrams.

In non-Abelian gauge theories however, the aforementioned stronger version of the OT does not hold in general. The reason is that unlike their scalar or Abelian counterparts, the conventional self-energies, vertex and boxes are gauge dependent. Noticeable exceptions of gauges in nonAbelian theories where the OT holds graph by graph are the axial gauges in the case of QCD, and the "unitary" gauge in theories with massive gauge bosons. Nevertheless, it is known that in both cases the Green's functions obtained have bad renormalization properties. In the case of QCD, the gluon self-energy computed within the axial gauges is not multiplicatively renormalizable [16]. Furthermore, spurious infrared divergences appear in the Feynman parameter integrations, which are artifacts and cancel out only when physical quantities are computed [31]. As for the unitary gauges, it is well-known that the two-, three-, and four-point functions computed in this scheme are non-renormalizable. As has been demonstrated in a series of papers $[18,19,22]$ however, a strong version of the OT very analogous to Eq. (3.1) can be realized in the context of non-Abelian gauge theories at one loop, if the amplitudes are rearranged according to the PT algorithm.

Specifically, let us apply the PT on both sides of Eq. (3.1): The PT rearrangement of the tree-level cross sections appearing in the RHS gives rise to new process-independent (selfenergy-like) parts, which are equal to the imaginary part of the effective self-energies obtained by the application of the PT on the one-loop expression for the amplitude $\langle a|T| b\rangle$ on the LHS. The same result is true for the vertex- and box-like parts, defined by the PT on either side of Eq. (3.1). In other 


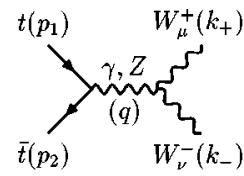

(a)

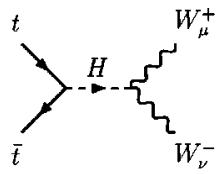

(b)

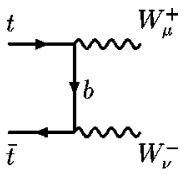

(c)
FIG. 3. Feynman diagrams pertaining to the process $t \bar{t}$ $\rightarrow W^{+} W^{-}$.

words, effective sub-amplitudes obtained after the application of the PT satisfy the OT individually, e.g.,

$$
\operatorname{Im}\left(\langle a|T| a\rangle_{\mathrm{PT}}^{j}\right)=\frac{1}{2} \sum_{f} \int\left(\langle f|T| a\rangle\langle f|T| a\rangle^{*}\right)_{\mathrm{PT}}^{j}
$$

where the subscript "PT', indicates that the PT rearrangement has been carried out, and the index $j=S, V, B$, distinguishes between effective self-energy, vertex, and boxes, respectively.

Turning to a specific example involving the Higgs boson, let us apply the previous arguments to the case of the process $t \bar{t} \rightarrow t \bar{t}$. At the tree-level this process can be mediated by a photon, a $Z$ boson, and a possibly resonant $H$ scalar. We focus on the sub-amplitude which contains two intermediate $W$ bosons. In that case the OT yields

$$
\operatorname{Im}\langle t \bar{t}|T| t \bar{t}\rangle=\frac{1}{2} \int d X_{\mathrm{LIPS}}\left\langle t \bar{t}|T| W^{+} W^{-}\right\rangle\left\langle W^{+} W^{-}|T| t \bar{t}\right\rangle *,
$$

where the Lorentz-invariant phase-space (LIPS) measure is defined as

$$
\begin{aligned}
\int d X_{\mathrm{LIPS}}= & \frac{1}{(2 \pi)^{2}} \int d^{4} k_{1} \int d^{4} k_{2} \delta_{+}\left(k_{1}^{2}-M_{W}^{2}\right) \\
& \times \delta_{+}\left(k_{2}^{2}-M_{W}^{2}\right) \delta^{(4)}\left(q-k_{1}-k_{2}\right),
\end{aligned}
$$

and $\delta_{+}\left(k^{2}-m^{2}\right) \equiv \theta\left(k^{0}\right) \delta\left(k^{2}-m^{2}\right)$. We now introduce the abbreviations $\mathcal{M}=\left\langle t\left(p_{1}\right) \bar{t}\left(p_{2}\right)|T| t\left(p_{1}\right) \bar{t}\left(p_{2}\right)\right\rangle \quad$ and $\mathcal{T}$ $=\left\langle t\left(p_{1}\right) \bar{t}\left(p_{2}\right)|T| W^{+}\left(k_{+}\right) W^{-}\left(k_{-}\right)\right\rangle$, and focus on the RHS of Eq. (3.3). Diagrammatically, the amplitude $\mathcal{T}$ consists of two distinct parts: an $s$-channel amplitude, $\mathcal{T}_{s \mu \nu}$, which is given in Figs. 3(a) and 3(b), and a $t$-channel amplitude, $\mathcal{T}_{t \mu \nu}$, which depends on the $b$ quark propagator, as shown in Fig. $3(c)$. The subscript " $s$ " and " $t$ " refers to the corresponding Mandelstam variables, i.e., $s=q^{2}=\left(p_{1}+p_{2}\right)^{2}=\left(k_{+}+k_{-}\right)^{2}$, and $t=\left(p_{1}-k_{+}\right)^{2}=\left(p_{2}-k_{-}\right)^{2}$. $\mathcal{T}_{s \mu \nu}$ can be further decomposed into two different $s$-exchange amplitudes: one mediated by a Higgs boson, denoted by $\mathcal{T}_{s \mu \nu}^{H}$, and one mediated by the two neutral gauge bosons $\gamma$ and $Z$, denoted by $\mathcal{T}_{s \mu \nu}^{V}$, with $V=\gamma, Z$. The explicit form of the above amplitudes reads:

$$
\begin{aligned}
\mathcal{T}_{s \mu \nu}^{V}= & -\frac{g_{w}^{2}}{2} \sum_{V=\gamma, Z} \bar{v}\left(p_{2}\right) \gamma_{\rho}\left(g_{v}^{V}+g_{a}^{V} \gamma_{5}\right) u\left(p_{1}\right) \\
& \times U_{V}^{\rho \lambda}(q) \Gamma_{\lambda \mu \nu}\left(q, k_{+}, k_{-}\right),
\end{aligned}
$$

$$
\begin{aligned}
\mathcal{T}_{s \mu \nu}^{H} & =\frac{g_{w}^{2}}{2} m_{t} \bar{v}\left(p_{2}\right) u\left(p_{1}\right) \Delta_{H}(q) g_{\mu \nu}, \\
\mathcal{T}_{t}^{\mu \nu} & =-\frac{g_{w}^{2}}{2} \bar{v}\left(p_{2}\right) \gamma^{\nu} P_{L} \frac{1}{p_{1}-k_{+}-m_{b}} \gamma^{\mu} P_{L} u\left(p_{1}\right),
\end{aligned}
$$

with $\Delta_{H}(q)=\left(q^{2}-M_{H}^{2}\right)^{-1}, \quad g_{v}^{\gamma}=4 \sin ^{2} \theta_{w} / 3, \quad g_{a}^{\gamma}=0, \quad g_{v}^{Z}$ $=1 / 2-g_{v}^{\gamma}, g_{a}^{Z}=-1 / 2$, and

$$
\begin{aligned}
\Gamma_{\lambda \mu \nu}\left(q, k_{+}, k_{-}\right)= & \left(k_{-}-k_{+}\right)_{\lambda} g_{\mu \nu}-\left(q+k_{-}\right)_{\mu} g_{\lambda \nu} \\
& +\left(q+k_{+}\right)_{\nu} g_{\mu \lambda} .
\end{aligned}
$$

In Eq. (3.5), $U_{Z}^{\rho \lambda}(q)$ denotes the propagator of the $Z$ boson in the unitary gauge, and $U_{\gamma}^{\rho \lambda}(q)$ is the photon propagator in an arbitrary gauge. The gauge dependence of the photon is trivially canceled, as soon as $\mathcal{T}_{s \mu \nu}^{V}$ is contracted with the polarization vectors of the $W$ bosons. With the definitions given above, the RHS of Eq. (3.3) becomes

$$
\begin{aligned}
\operatorname{Im} \mathcal{M}= & \mathcal{T}_{\mu \nu} Q^{\mu \rho}\left(k_{+}\right) Q^{\nu \sigma}\left(k_{-}\right) \mathcal{T}_{\rho \sigma}^{*} \\
= & {\left[\mathcal{T}_{s \mu \nu}^{V}+\mathcal{T}_{s \mu \nu}^{H}+\mathcal{T}_{t \mu \nu}\right] Q^{\mu \rho}\left(k_{+}\right) Q^{\nu \sigma}\left(k_{-}\right) } \\
& \times\left[\mathcal{T}_{s \rho \sigma}^{V}+\mathcal{T}_{s \rho \sigma}^{H}+\mathcal{T}_{t \rho \sigma}\right]^{*}
\end{aligned}
$$

where

$$
Q^{\mu \nu}(k)=-g^{\mu \nu}+\frac{k^{\mu} k^{\nu}}{M_{W}^{2}}
$$

is the $W$ polarization tensor. Obviously, $k^{\mu} Q_{\mu \nu}(k)=0$, when $k^{2}=M_{W}^{2}$. Furthermore, in Eq. (3.9), we omit the integration measure $1 / 2 \int d X_{\text {LIPS }}$.

Since our main interest lies in the Higgs-boson-mediated interaction contained in the transition $t \bar{t} \rightarrow t \bar{t}$, we wish to isolate the part which depends on the Higgs boson. In doing so particular care is needed, because, despite appearances, the $t$-channel amplitude $\mathcal{T}_{t}^{\mu \nu}$ contains contributions which are related to the Higgs-boson interaction. These contributions emerge by virtue of the following WI:

$$
\begin{aligned}
\frac{k_{+}^{\mu} k_{-}^{\nu}}{M_{W}^{2}} \mathcal{T}_{t \mu \nu} & =\mathcal{T}_{P}^{H}+\cdots, \\
\mathcal{T}_{P}^{H} & =-\frac{g_{w}^{2}}{4} \frac{m_{t}}{M_{W}^{2}} \bar{v}\left(p_{2}\right) u\left(p_{1}\right),
\end{aligned}
$$

shown schematically in Fig. 2. The above WI is triggered by the longitudinal momenta $k_{+}^{\mu}$ and $k_{-}^{\nu}$, originating from the polarization tensors $Q^{\mu \rho}\left(k_{+}\right)$and $Q^{\nu \sigma}\left(k_{-}\right)$, respectively. The ellipses in Eq. (3.11) denote additional contributions which are not related to the Higgs boson, i.e., their coupling to the external fermions is not proportional to $m_{t}$. Notice that the combined action of both $k_{+}^{\mu}$ and $k_{-}^{\nu}$ is necessary, in order for the piece related to the Higgs boson to appear. 
We then proceed to carry out the multiplication on the RHS of Eq. (3.9) (we suppress Lorentz indices). To begin with, the term $\mathcal{T}_{s}^{V} Q\left(k_{+}\right) Q\left(k_{-}\right) \mathcal{T}_{s}^{V *}$ has no dependence on the Higgs boson, and we can discard it. In addition, the terms $\mathcal{T}_{s}^{H} Q\left(k_{+}\right) Q\left(k_{-}\right) \mathcal{T}_{s}^{V *}$ and $\mathcal{T}_{s}^{V} Q\left(k_{+}\right) Q\left(k_{-}\right) \mathcal{T}_{t}$ give Higgsboson related pieces, which are however antisymmetric under the exchange $k_{+} \leftrightarrow k_{-}$, and therefore vanish upon the symmetric phase-space integration. This may be readily verified, if one employs the following two identities:

$$
\begin{gathered}
\Gamma_{\lambda \mu \nu}\left(q, k_{+}, k_{-}\right) Q^{\mu \rho}\left(k_{+}\right) Q_{\rho}^{\nu}\left(k_{-}\right)=\left[\frac{\left(q^{2}\right)^{2}}{4 M_{W}^{4}}-3\right]\left(k_{+}-k_{-}\right)_{\lambda}, \\
k_{+}^{\mu} k_{-}^{\nu} \Gamma_{\lambda \mu \nu}\left(q, k_{+}, k_{-}\right)=\frac{q^{2}}{2}\left(k_{+}-k_{-}\right)_{\lambda} .
\end{gathered}
$$

This last result is in agreement with earlier observations [25], that any non-vanishing $Z H$ transition would lead to $C P$ violation, and therefore, it should be absent in a $C P$-invariant theory, such as the bosonic part of the bare Lagrangian of the SM. Finally, the part of Eq. (3.9) related to the Higgs boson reads:

$$
\begin{aligned}
\operatorname{Im} \mathcal{M}_{\mathrm{Higgs}}= & Q\left(k_{+}\right) Q\left(k_{-}\right) \mathcal{T}_{s}^{H} \mathcal{T}_{s}^{H *}+\left[Q\left(k_{+}\right) Q\left(k_{-}\right)\right. \\
& \left.\times\left(\mathcal{T}_{s}^{H} \mathcal{T}_{t}^{*}+\mathcal{T}_{t} \mathcal{T}_{s}^{H *}+\mathcal{T}_{t} \mathcal{T}_{t}^{*}\right)\right]_{\mathrm{Higgs}} .
\end{aligned}
$$

This last expression will be now separated into two distinct pieces as follows:

$$
\operatorname{Im} \mathcal{M}_{\text {Higgs }}=\operatorname{Im} \hat{\mathcal{M}}_{\text {self }}^{H}+\operatorname{Im} \hat{\mathcal{M}}_{\text {vert }}^{H},
$$

i.e., a universal, self-energy-like piece, $\operatorname{Im} \hat{\mathcal{M}}_{\text {self }}^{H}$, which does not depend on the propagator of the $b$ quark, and a vertexlike piece $\operatorname{Im} \hat{\mathcal{M}}_{\text {vert }}^{H}$, which explicitly contains the $b$ quark propagator. The propagator-like contribution may be written as

$$
\begin{aligned}
\operatorname{Im} \hat{\mathcal{M}}_{\text {self }}^{H}= & \mathcal{T}_{s \mu \nu}^{H} Q^{\mu \lambda}\left(k_{+}\right) Q^{\nu \rho}\left(k_{-}\right) \mathcal{T}_{s \lambda \rho}^{H *}+\left(\frac{1}{2} g^{\mu \nu}+\frac{k_{+}^{\mu} k_{-}^{\nu}}{M_{W}^{2}}\right) \\
& \times\left(\mathcal{T}_{s \mu \nu}^{H} \mathcal{T}_{P}^{H *}+\mathcal{T}_{P}^{H} \mathcal{T}_{s \mu \nu}^{H *}\right)+\mathcal{T}_{P}^{H} \mathcal{T}_{P}^{H *} .
\end{aligned}
$$

The closed expressions for the terms on the RHS of Eq. (3.16) are as follows:

$$
\begin{aligned}
\mathcal{T}_{s \mu \nu}^{H} Q^{\mu \lambda}\left(k_{+}\right) Q^{\nu \rho}\left(k_{-}\right) \mathcal{T}_{s \lambda \rho}^{H *} \\
=\left(\frac{g m_{t}}{2 M_{W}}\right)^{2} \bar{v}\left(p_{2}\right) u\left(p_{1}\right) \Delta_{H}(q)\left(\frac{g^{2}}{4}\right) \\
\quad \times\left[\left(q^{2}\right)^{2}-4 q^{2} M_{W}^{2}+12 M_{W}^{4}\right] \Delta_{H}(q) \bar{u}\left(p_{1}\right) v\left(p_{2}\right),
\end{aligned}
$$

$$
\begin{aligned}
& \left(\frac{1}{2} g^{\mu \nu}+\frac{k_{+}^{\mu} k_{-}^{\nu}}{M_{W}^{2}}\right) \mathcal{T}_{s \mu \nu}^{H} \mathcal{T}_{P}^{H *}+\text { c.c. } \\
& =-\left(\frac{g m_{t}}{2 M_{W}}\right)^{2} \bar{v}\left(p_{2}\right) u\left(p_{1}\right) \Delta_{H}(q)\left(\frac{g^{2}}{4}\right)\left(q^{2}-M_{H}^{2}\right) \\
& \quad \times\left(q^{2}+2 M_{W}^{2}\right) \Delta_{H}(q) \bar{u}\left(p_{1}\right) v\left(p_{2}\right), \\
& \mathcal{T}_{P}^{H} \mathcal{T}_{P}^{H *}=\left(\frac{g m_{t}}{2 M_{W}}\right)^{2} \bar{v}\left(p_{2}\right) u\left(p_{1}\right) \Delta_{H}(q)\left(\frac{g^{2}}{4}\right) \\
& \times\left(q^{2}-M_{H}^{2}\right)^{2} \bar{u}\left(p_{1}\right) v\left(p_{2}\right),
\end{aligned}
$$

where the abbreviation c.c. stands for complex conjugation. After adding the above propagator-like contributions and carrying out the two $W$-boson phase-space integration, we define the imaginary part of the effective PT self-energy for the Higgs boson in the conventional way, i.e., as the part of the above amplitude which is sandwiched between the two bare Higgs boson propagators $\Delta_{H}(q)$. In this way we obtain

$$
\begin{aligned}
\operatorname{Im} \hat{\Pi}_{(W W)}^{H H}\left(q^{2}\right)= & \frac{\alpha_{w}}{16} \frac{M_{H}^{4}}{M_{W}^{2}}\left(1-\frac{4 M_{W}^{2}}{q^{2}}\right)^{1 / 2} \\
& \times\left[1+4 \frac{M_{W}^{2}}{M_{H}^{2}}-4 \frac{M_{W}^{2}}{M_{H}^{4}}\left(2 q^{2}-3 M_{W}^{2}\right)\right] .
\end{aligned}
$$

Notice the crucial cancellation of the $\left(q^{2}\right)^{2}$ terms; had such terms survived, they would have given rise to a running width which would grossly contradict the ET (see also discussion in Sec. VI). Equation (3.20) is in agreement with the result reported in [25].

We can now easily establish contact with the results of the previous section. Starting from Eq. (2.9), we can arrive at Eq. (3.20) by using the following relation:

$$
\begin{aligned}
\frac{1}{16 \pi^{2}} & \operatorname{Im} B_{0}\left(q^{2}, m_{1}^{2}, m_{2}^{2}\right) \\
= & -\frac{1}{16 \pi^{2}} \operatorname{Im}\left\{\int _ { 0 } ^ { 1 } d x \operatorname { l n } \left[m_{1}^{2} x+m_{2}^{2}(1-x)\right.\right. \\
& \left.\left.-q^{2} x(1-x)\right]\right\} \\
= & \theta\left[q^{2}-\left(m_{1}+m_{2}\right)^{2}\right] \frac{1}{16 \pi q^{2}} \lambda^{1 / 2}\left(q^{2}, m_{1}^{2}, m_{2}^{2}\right) \\
= & \frac{1}{2} \int d X_{\mathrm{LIPS}},
\end{aligned}
$$

with $\lambda(x, y, z)=(x-y-z)^{2}-4 y z$. Of course, for the case at hand, we have $m_{1}=m_{2}=M_{W}$. Conversely, we can recover from Eq. (3.20) the on-shell renormalized result of Eq. (2.9) by means of a twice-subtracted (on shell) dispersion relation $[19,22]$. 

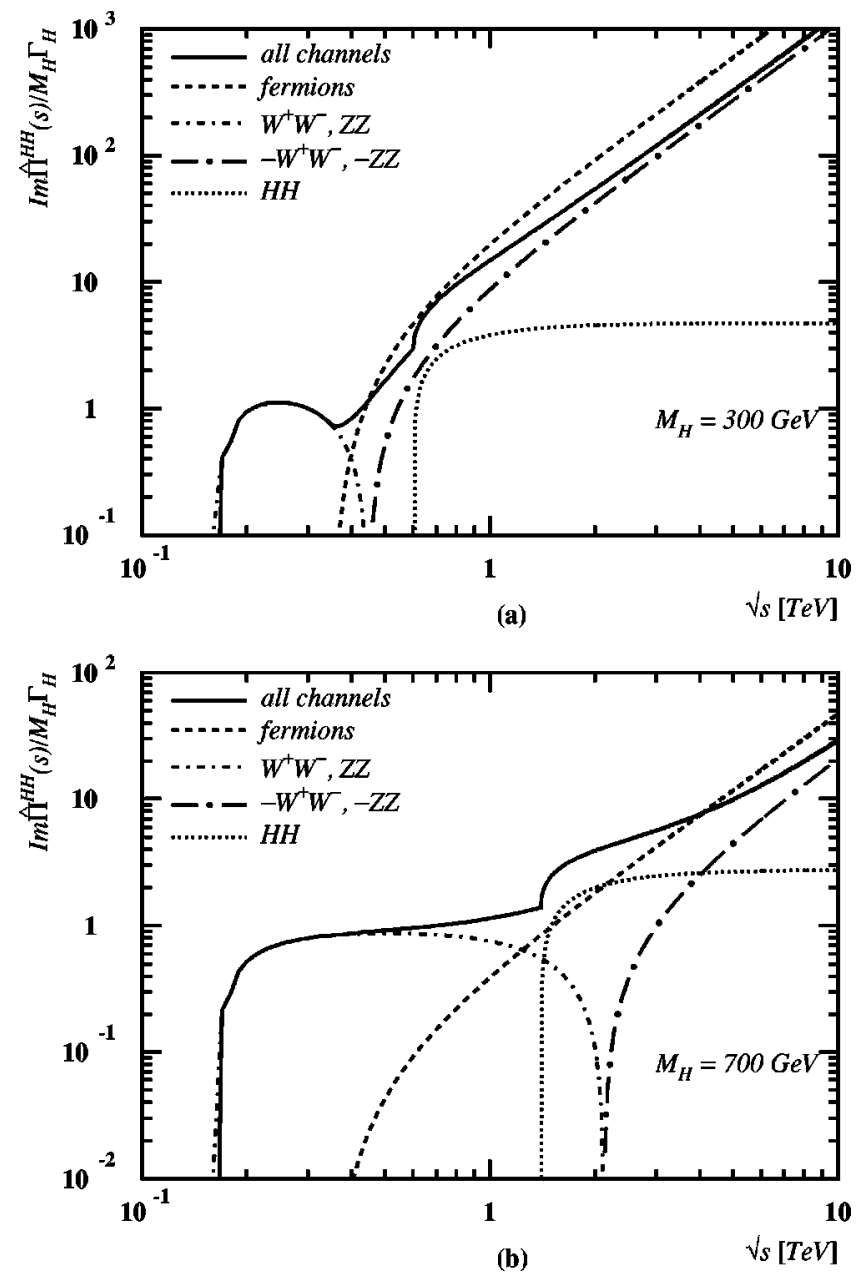

FIG. 4. Dependence of $\operatorname{Im} \hat{\Pi}^{H H}(s) / M_{H} \Gamma_{H}$ on $\sqrt{s}$ for individual intermediate states.

The contribution to the PT Higgs self-energy, which comes from two intermediate $Z$ bosons, may be obtained in an analogous way. For definiteness, in Fig. 4 we plot separately the dependence of all the kinematic channels involved in $\operatorname{Im} \hat{\Pi}^{H H}(s)$ as a function of the center-of-mass (c.m.) energy $\sqrt{s}$. The solid line corresponds to the total effect of all intermediate states. In Fig. 4(a), we have displayed the results of a light Higgs scenario with a mass $M_{H}=300 \mathrm{GeV}$, whereas predictions obtained for a heavy Higgs with $M_{H}$ $=700 \mathrm{GeV}$ are presented in Fig. 4(b). Notice that the absorptive part of the bosonic channels $\operatorname{Im} \hat{\Pi}_{(V V)}^{H H}(s)=\operatorname{Im} \hat{\Pi}_{(W W)}^{H H}(s)$ $+\operatorname{Im} \hat{\Pi}_{(Z Z)}^{H H}(s)$, represented by a dash-dotted line in both plots, turns negative far above the resonant point $s=M_{H}^{2}$, as can be readily deduced from the closed expressions given in Eqs. (2.9) and (2.29). Specifically, $\operatorname{Im} \hat{\Pi}_{(V V)}^{H H}(s)$ turns negative when $\sqrt{s}>430 \mathrm{GeV}$ for $M_{H}=300 \mathrm{GeV}$, and $\sqrt{s}>2 \mathrm{TeV}$ for $M_{H}=700 \mathrm{GeV}$. The dependence of $-\operatorname{Im} \hat{\Pi}_{(V V)}^{H H}(s)$ on $\sqrt{s}$ is indicated by a long-dash-dotted line. However, we must remark that the total absorptive part of the Higgs boson selfenergy stays always positive due to the large positive contribution of the heavy top quark $\left(m_{t}=170 \mathrm{GeV}\right)$. Thus, at c.m. energies $\sqrt{s} \gg M_{H}, \operatorname{Im} \hat{\Pi}^{H H}(s)$ has the following asymptotic behavior:

$$
\operatorname{Im} \hat{\Pi}^{H H}(s) \sim \frac{\alpha_{w} s}{8 M_{W}^{2}}\left(3 m_{t}^{2}-4 M_{W}^{2}-2 M_{Z}^{2}\right) .
$$

The fact that the bosonic contributions to the absorptive part of the Higgs-boson self-energy is negative at large $s$ is reminiscent of the PT gauge-boson self-energies in theories with asymptotic freedom, whose absorptive parts are also negative. For instance, the absorptive part of the PT (or BFM) gluon self-energy in quark-less QCD has the exact same feature, and, as a result, it does not admit the usual KällenLehmann spectral representation. By analogy, far above the resonant point, the resummed Higgs-boson propagator loses its meaning as a description of the $\mathrm{BW}$ dynamics of the unstable Higgs particle, but it rather serves as the "effective charge" of the universal Higgs-mediated part of the electroweak interaction. In Sec. V C, we will take a closer look at this issue.

In the following, we study the resonant behavior of the resummed Higgs-boson propagator

$$
\hat{\Delta}^{H}(s)=\left[s-M_{H}^{2}+\hat{\Pi}^{H H}(s)\right]^{-1},
$$

within different approaches. For example, $\hat{\Delta}^{H}(s)$ may occur in the process $t \bar{t} \rightarrow H^{*} \rightarrow t \bar{t}$. In Fig. 5, we display the dependence of the modulus of the resummed Higgs-boson propagator as a function of the c.m. energy $\sqrt{s}$. The solid line refers to the result obtained in the PT resummation approach, whereas the dashed, dotted and dash-dotted lines correspond to resumming Higgs self-energies in the BFG with $\xi_{Q}$ $=100,1000$, and in the unitary gauge, respectively. Notice the characteristic presence of unphysical thresholds in the BFG, which manifest themselves as artificial resonances. As can also be seen from Figs. 5(a) and 5(b) (for $M_{H}=300$ and $700 \mathrm{GeV}$, respectively), in the unitary gauge the width increases as $s^{2}$ and distorts the Higgs-boson lineshape. As a final remark, we point out that the usual description of unstable particles by means of a constant width approach, where $M_{H}^{2}-\hat{\Pi}^{H H}(s)$ is replaced by the complex pole $M_{H}^{2}$ $-i M_{H} \Gamma_{H}$ in $\hat{\Delta}^{H}(s)$ for any value of $s$, leads in the limit $s$ $\rightarrow 0$ to a non-vanishing $\operatorname{Im} \hat{\Delta}^{H}(s)$, and therefore violates the OT [18].

\section{RENORMALIZATION GROUP ANALYSIS}

The ultimate goal of this program is to provide a systematic framework for constructing physically meaningful Bornimproved approximations for resonant transition amplitudes. In doing so, we have mainly focused on gauge-invariance and unitarity, and shown how one can manifestly maintain such crucial properties even when resonant bosonic contributions are considered. In the next two sections we turn to another important property, namely the invariance of the Born amplitudes under the renormalization group. In particular, we will show explicitly that the amplitudes obtained by 

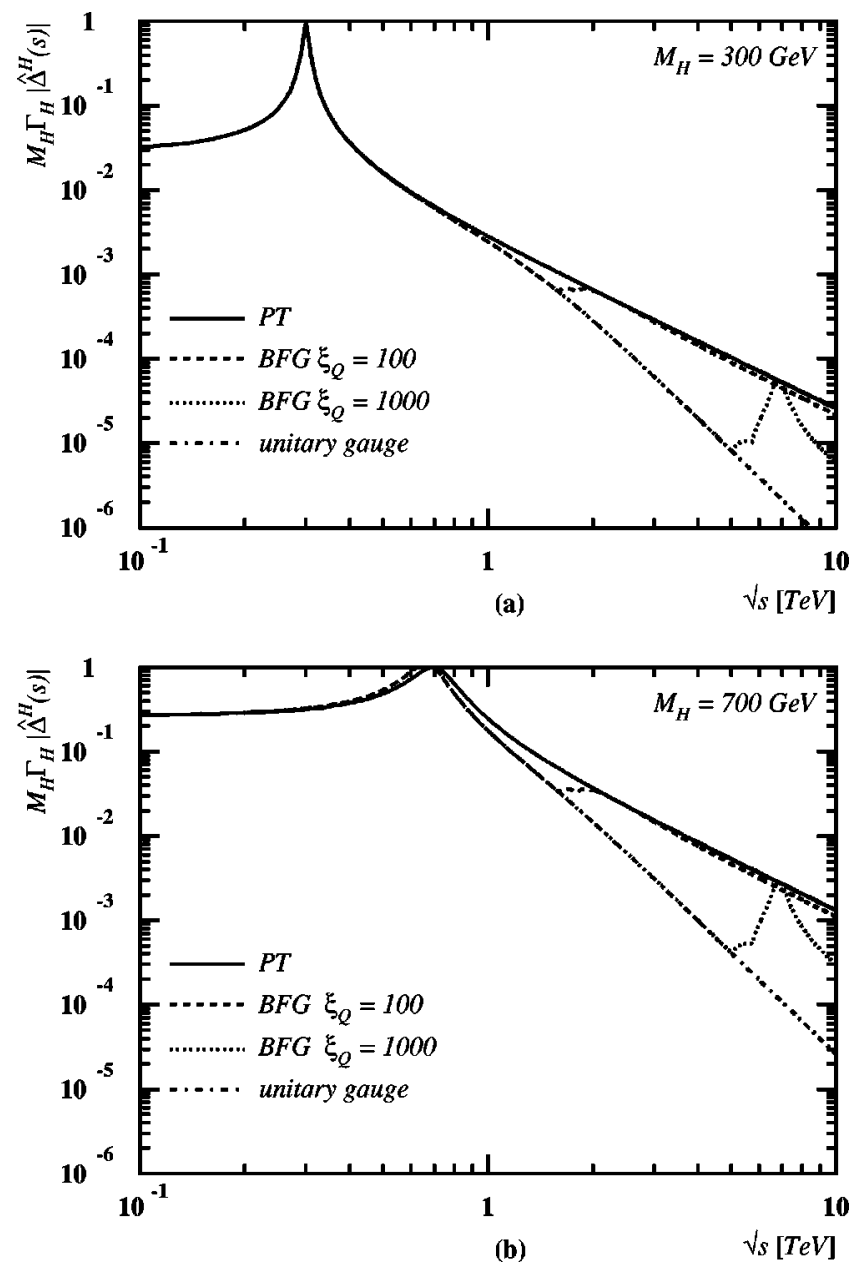

FIG. 5. $M_{H} \Gamma_{H}\left|\hat{\Delta}^{H}(s)\right|$ versus $\sqrt{s}$ in different resummation schemes.

our resummation method are built out of renormalizationgroup-invariant structures. Furthermore, we will demonstrate how one can generalize the effective charge, a familiar concept in the context of gauge bosons such as the $W$ and $Z$ bosons, to the case of the scalar Higgs boson. This scalar "effective charge", constitutes a common component in every Higgs-boson mediated process, regardless of the nature of incoming and outgoing states, and can thus be viewed as a universal, process-independent entity, intrinsic to the Higgs boson.

This section is organized as follows: We first review the concept of the effective charge in the context of QED; then we discuss its generalization to the case of a non-Abelian gauge theory, such as QCD. The crux of this analysis is that by virtue of the WI's present in gauge theories the effective charge is both invariant under the renormalization group and process-independent. At the end of this section we discuss a counter-example, i.e., the case of an asymptotically free scalar model in six space-time dimensions, and analyze the reasons which make the construction of an effective charge not possible. In particular, we explain why in this theory one cannot reconcile invariance under renormalization group and process independence. Interestingly enough, the construction of an effective charge in a scalar context becomes again pos- sible after adding, and subsequently breaking spontaneously, a global symmetry, as we will show at the end of this section.

\section{A. Effective charge in QED}

We start our discussion with the case of QED. The Abelian gauge symmetry of the theory gives rise to the fundamental WI: $\quad q_{\mu} \Gamma^{\mu, 0}(p, p+q)=S^{0-1}(p+q)-S^{0-1}(p)$, where $\Gamma^{\mu, 0}$ is the bare photon-electron vertex and $S^{0}(k)$ the dressed electron propagator. The above identity is valid both perturbatively to all orders, as well as non-perturbatively. The requirement that the renormalized vertex $\Gamma^{\mu}=Z_{1} \Gamma^{\mu, 0}$ and the renormalized self-energy $S=Z_{f}^{-1} S^{0}$ satisfy the same identity imposes the equality between the vertex renormalization constant $Z_{1}$ and the electron wave-function renormalization constant $Z_{f}$, namely $Z_{1}=Z_{f}$. As a result, the photon wave-function renormalization constant $Z_{A}$ and the charge renormalization constant $Z_{e}=Z_{1} Z_{2}^{-1} Z_{3}^{-1 / 2}$ are related by the following fundamental equation:

$$
Z_{e}=Z_{A}^{-1 / 2} \text {. }
$$

The unrenormalized photon self-energy is $\Pi_{\mu \nu}^{0}(q)$ $=\left(-q^{2} g_{\mu \nu}+q_{\mu} q_{\nu}\right) \Pi^{0}\left(q^{2}\right)$, where $\Pi^{0}\left(q^{2}\right)$ is a GFPindependent function to all orders in perturbation theory. After performing the standard Dyson summation, we obtain the dressed propagator between conserved external currents

$$
\Delta_{\mu \nu}^{0}(q)=\frac{-g_{\mu \nu}}{q^{2}\left[1+\Pi^{0}\left(q^{2}\right)\right]} .
$$

The above quantity is universal, in the sense that is process independent. We can now form the following RGI combination:

$$
R_{\mu \nu}^{e}\left(q^{2}\right) \equiv \alpha_{\mathrm{eff}}\left(q^{2}\right) \frac{-g_{\mu \nu}}{q^{2}}
$$

where

$$
\alpha_{\mathrm{eff}}\left(q^{2}\right)=\frac{\left(e^{0}\right)^{2}}{4 \pi} \frac{1}{1+\Pi^{0}\left(q^{2}\right)}=\frac{e^{2}}{4 \pi} \frac{1}{1+\Pi\left(q^{2}\right)}
$$

The last equality in Eq. (4.4) can be readily obtained if one uses the relations between renormalized and unrenormalized parameters: $e^{2}=\left(Z_{f}^{2} Z_{A} / Z_{1}^{2}\right)\left(e^{0}\right)^{2}, 1+\Pi\left(q^{2}\right)=Z_{A}[1$ $\left.+\Pi^{0}\left(q^{2}\right)\right]$, and $Z_{1}=Z_{f}$. For $q^{2} \gg m_{e}^{2}, \alpha_{\text {eff }}\left(q^{2}\right)$ coincides with the one-loop running coupling of the theory. We must remark that the effective charge has a non-trivial dependence on the masses of the particles appearing in the quantum loops, which allows its reconstruction from physical amplitudes [22]. In general, the transition amplitude of a QED process, such as $e^{+} e^{-} \rightarrow e^{+} e^{-}$, consists of two RGI combinations: a process-independent one, namely the effective QED charge defined above, and a process-dependent one, namely the sum of vertex and box diagrams. 


\section{B. Effective charge in QCD}

In non-Abelian gauge theories the crucial equality $Z_{1}$ $=Z_{f}$ does not hold in general. Furthermore, in contrast to the photon case, the gluon vacuum polarization depends on the GFP, already at one loop order. These facts make the nonAbelian generalization of the QED concept of the effective charge non-trivial. The possibility of defining an effective charge for QCD in the framework of the PT was discussed first by Cornwall [15], and was further investigated in a series of recent papers $[18,32,33]$.

The PT rearrangement of physical amplitudes gives rise to a GFP-independent effective gluon self-energy, and restores at the same time the equalities

$$
\hat{Z}_{1}=\hat{Z}_{f}, \quad \hat{Z}_{g}=\hat{Z}_{A}^{-1 / 2},
$$

where the carets denote the corresponding renormalization constants in the PT, and $g$ is the QCD coupling. Having restored QED-like WI's and GFP independence, and using the additional fact that the one-loop PT self-energy is process-independent [34] and can be Dyson-resummed to all orders $[18,33]$, the construction of the universal RGI combination and the corresponding QCD effective charge is immediate. We have

$$
\hat{R}_{\mu \nu}^{g}\left(q^{2}\right) \equiv \alpha_{s, \mathrm{eff}}\left(q^{2}\right) \frac{-g_{\mu \nu}}{q^{2}}
$$

where

$$
\alpha_{s, \mathrm{eff}}\left(q^{2}\right)=\frac{\left(g^{0}\right)^{2}}{4 \pi} \frac{1}{1+\hat{\Pi}^{0}\left(q^{2}\right)}=\frac{g^{2}}{4 \pi} \frac{1}{1+\hat{\Pi}\left(q^{2}\right)}
$$

It is interesting to note that in the BFM formulation of QCD the Green's functions satisfy by construction QED WI's, to all orders in perturbation theory. On the other hand, the BFM Green's functions still depend on the GFP $\xi_{Q}$. In the case of the gluon vacuum polarization this dependence on $\xi_{Q}$ is trivial, since it does not affect the prefactor of the leading logarithm, and is just an additive constant. This constant may be considered as an arbitrariness in the renormalization scheme, and will hence disappear when forming the schemeindependent RGI quantity given in Eq. (4.6). This is however not true in the case of massive gauge bosons; there, the dependence on the GFP cannot be removed by means of an appropriate choice of renormalization scheme.

\section{The scalar case}

The ability to define a process-independent RGI quantity is not a common characteristic of all field theories; for example this is not what happens in the case of pure scalar theories. However, if the scalar theory is spontaneously broken, then a RGI effective charge may be defined for the Higgs boson, which is inversely proportional to its vacuum expectation value (VEV). In the following, we shall examine both situations.

Let us first study $\left(\phi^{3}\right)_{6}$, i.e., scalar $\phi^{3}$ in six space-time dimensions. The theory is asymptotically free, gauge invari- ance is of course not an issue, and just as in QED, the OT holds for individual Feynman diagrams, and the self-energy can be formally Dyson-resummed. However, unlike QED, if one was to use this formally resummed self-energy inside a tree-level amplitude, the resulting expression would not be renormalization group invariant. The reason is that there is no QED-like WI enforcing the equality between vertex and wave-function renormalization. As a result of that, it is only after the vertex correction have been included that the resulting combination becomes a RGI combination. The drawback of this is that the inclusion of the vertices introduces processdependence. In other words, in the $\left(\phi^{3}\right)_{6}$ case it is not possible to construct a RGI quantity which is, at the same time, process independent, i.e., one cannot reconcile process independence and renormalization group invariance [35].

To see this in detail, let us study the Veltman model [36] at $d=6$ instead of $d=4$. This theory contains a light scalar, $\phi$, and a heavy scalar, $\Phi$, with a mass $M_{\Phi}>2 M_{\phi}$. The heavy scalar decays into two $\phi$ 's, via the interaction term in the Lagrangian

$$
\mathcal{L}_{i n t}=\frac{\lambda}{2} \phi^{2} \Phi
$$

where $\lambda$ is a non-zero coupling constant. The wave-function renormalization constants $Z_{\phi}$ and $Z_{\Phi}$, corresponding to the fields $\phi$ and $\Phi$, respectively, and the vertex renormalization $Z_{\phi^{2} \Phi}$ have been calculated in the minimal subtraction scheme [37]. They are

$$
Z_{\phi}=Z_{\Phi}=1+\left(\frac{1}{6}\right) \frac{g^{2}}{64 \pi^{3} \epsilon}, \quad Z_{\lambda}=1+\frac{g^{2}}{64 \pi^{3} \epsilon} .
$$

Clearly, one has $Z_{\Phi} \neq Z_{\lambda}$. Of course, since the pole terms of $Z_{\Phi}$ and $Z_{\lambda}$ are different, the above inequality will be true in any other renormalization scheme. Consequently, for the charge renormalization $Z_{\lambda}$, defined by the equation $\lambda^{0}$ $=Z_{\lambda} \lambda$, we have that $Z_{\lambda}=Z_{\phi^{2} \Phi} Z_{\phi}^{-1} Z_{\Phi}^{-1 / 2}=Z_{\phi^{2} \Phi} Z_{\Phi}^{-3 / 2}$ $\neq Z_{\Phi}^{-1}$. As a result, for the combination $\left(\lambda^{0}\right)^{2} \Delta^{0}(s)$, which is the direct analog of the QED effective charge, we have that $\left(\lambda^{0}\right)^{2} \Delta^{0}(s) \neq \lambda^{2} \Delta(s)$. Therefore, in order to arrive at a RGI expression, the vertex correction must be supplemented. So, the combination

$$
\phi^{0} \phi^{0} \Gamma^{0} \Delta^{0} \Gamma^{0} \phi^{0} \phi^{0}=\phi \phi \Gamma \Delta \Gamma \phi \phi
$$

is a RGI quantity, but unlike the QED case, it cannot be written as the product of a process-independent and a process-dependent part, which are individually RGI.

To explore further how the process-dependence enters, it is instructive to add yet another set of scalar fields $\chi$, such that $M_{\Phi}>2 M_{\chi}>2 M_{\phi}$, and an extra interaction term

$$
\mathcal{L}_{\text {int }}^{\prime}=\frac{g}{2} \chi^{2} \Phi
$$

where $g$ is another non-zero coupling constant, with $g \neq \lambda$ in general. We will ignore for simplicity additional interaction terms such as $\phi^{3}, \chi^{3}, \phi^{2} \chi, \chi^{2} \phi$. Several of them may be eliminated by imposing an extra mirror symmetry of the type 
$\phi \rightarrow-\phi$ and $\chi \rightarrow-\chi$; in any case the presence of such terms does not alter our conclusions qualitatively [38].

In order to mimic gauge theories, we next set $g=\lambda$. Let us consider two different processes, $\phi \phi \rightarrow \phi \phi$ and $\chi \chi$ $\rightarrow \chi \chi$, both mediated by an $s$-channel resonant $\Phi$. The RGI quantities for the two processes are $\phi \phi \Gamma^{\phi^{2} \Phi}\left(s, M_{\Phi}, M_{\phi}\right) \Delta(s) \Gamma^{\phi^{2} \Phi}\left(s, M_{\Phi}, M_{\phi}\right) \phi \phi \quad$ and $\chi \chi \Gamma^{\chi^{2} \Phi}\left(s, M_{\Phi}, M_{\chi}\right) \Delta(s) \Gamma \chi^{2} \Phi\left(s, M_{\Phi}, M_{\chi}\right) \chi \chi$, where we have explicitly displayed the dependence of the vertices $\Gamma^{\phi^{2} \Phi}$ and $\Gamma^{\chi^{2} \Phi}$ on the masses. It is now easy to see that the process dependence enters through the simple fact that $\Gamma^{\phi^{2}} \Phi$ depends on $M_{\phi}$ but not on $M_{\chi}$, whereas the reverse is true for $\Gamma^{\chi^{2} \Phi}{ }^{2}$ Evidently, there is no RGI quantity common in these two amplitudes.

Let us now consider a four-dimensional $\Phi^{4}$ scalar theory which has a $\mathrm{U}(1)$ global invariance and includes a fermion $f$. The fermion $f$ is introduced in order to prevent the scalar theory from being super-renormalizable, so that one is able to study non-trivial renormalization effects. The part of the Lagrangian related to the Higgs potential of the model has the form

$$
\mathcal{L}_{V}=\mu^{2} \Phi^{*} \Phi+\lambda\left(\Phi^{*} \Phi\right)^{2} .
$$

The interaction of the scalar $\Phi$ to the fermion $f$ is given by

$$
\mathcal{L}_{\text {int }}=g \Phi \bar{f}_{L} f_{R}+\text { H.c. }
$$

The global U(1) symmetry of $\mathcal{L}_{V}$ in Eq. (4.12) breaks down spontaneously and the resulting theory resembles the ungauged SM, where the fermion $f$ may represent for example the top quark. Specifically, the field $\Phi$ must be expanded around its VEV, i.e., $\Phi=(v+H+i J) / \sqrt{2}$, where the field $H$ is a $C P$-even Higgs boson with mass $M_{H}=\sqrt{2} \mu$ and $J$ is the massless $C P$-odd Goldstone boson associated with the breaking of the continuous U(1) symmetry. After the SSB of the $\mathrm{U}(1)$ symmetry, the fermion $f$ acquires a mass $m_{f}$ $=g v / \sqrt{2}$. If $M_{H}>2 m_{f}$, then the decay process $H \rightarrow f \bar{f}$ is kinematically allowed and the Higgs boson becomes an unstable particle.

Beyond the Born approximation, the wave-function renormalization of the Higgs field $Z_{\Phi}^{1 / 2}$ renders the VEV $\left\langle\Phi^{0}\right\rangle$ $\equiv v^{0} \mathrm{UV}$ finite, $v i z ., v^{0}=Z_{\Phi}^{1 / 2}(v+\delta v)$, with vanishing divergent part $(\delta v)^{\mathrm{div}}=0$. As a consequence, the expression $\Delta^{0}(s) /\left(v^{0}\right)^{2}$, involving the resummed Higgs-boson propagator is RGI, i.e.,

$$
\frac{1}{\left(v^{0}\right)^{2}} \Delta^{0}(s)=\frac{1}{v^{2}} \Delta(s)
$$

It is then obvious that the VEV of the field $\Phi$ in a SSB scalar theory plays an instrumental role in defining a RGI effective charge for the Higgs interactions, very much in analogy to the QED and QCD cases discussed earlier. The Higgs field

\footnotetext{
${ }^{2}$ It is elementary to verify that the functional dependence of the vertex functions on the respective masses is non-trivial.
}

couples universally to matter with a "charge" inversely proportional to its VEV in the symmetric U(1) limit. If one now wishes to embed this scalar SSB model into a gauge theory, the situation becomes more involved. In fact, within conventional gauge-fixing schemes such as $R_{\xi}$ gauges, $\delta v$ is not UV finite and hence, Eq. (4.14) does no longer hold. In the next section, we shall discuss the possibility of identifying RGI effective charges for the gauge and Higgs bosons in the electroweak sector of the SM.

\section{EFFECTIVE CHARGES IN THE ELECTROWEAK SECTOR OF THE SM}

In the previous section we established in detail the conditions which enable the construction of process-independent RGI combinations for the gauge bosons of both Abelian and non-Abelian theories (QED and QCD, respectively). In this section we extend this analysis to the electroweak sector of the SM. First, we review how the construction of effective charges associated to the gauge bosons of the theory is possible by virtue of the WI's relating the PT effective $n$-point functions [22]. Furthermore, we discuss for the first time various subtleties related to the definition of the $W$ and $Z$ effective charges, which originate from the fact that the corresponding gauge bosons are unstable. We then turn to the case of the Higgs boson and examine the possibility of constructing a process-independent RGI quantity for the case of the Higgs boson. The answer to this question is by no means obvious, since the Higgs boson results from the SSB of the gauge group $\mathrm{SU}(2)_{L} \otimes \mathrm{U}(1)_{Y}$, and gauge-fixing and ghost terms spoil in general the equality (4.14). However, it turns out that because of the PT WI's, it is possible to construct a Higgs-boson "effective charge," in direct analogy to the gauge boson case.

\section{A. The PT Ward identities of the SM}

It is well-known that in the PT effective $n$-point functions satisfy (at least at one loop) naive, tree-level like WI's, as happens in QED. This is to be contrasted to the conventional $n$-point functions, which in general satisfy Slavnov-Taylor identities, which involve the Green's functions of the unphysical ghosts of the theory. The PT WI's are a direct consequence of the requirement that the S-matrix be GFP independent. The PT WI's for the electroweak sector of the SM have been derived in $[26,18]$. In fact, based on these WI's, it is possible to prove a stronger version of this GFP independence of the S-matrix: The S-matrix satisfies the "dual gauge-fixing property," which states that one is free to choose different GFP's for the gauge bosons inside and outside the quantum loops [39]. The above property is intrinsic to the S-matrix, and is not linked to any special gauge-fixing procedure. Its derivation is based on the observation that the PT rearrangement gives rise to one-loop $n$-point functions for which all dependence on the GFP stemming from the gauge bosons inside the quantum loops has disappeared, regardless of the choice of the GFP for the gauge bosons outside the loops. For the final cancellation of this latter gauge dependence to go through, the $n$-point functions constructed via the PT must satisfy tree-level WI's. Explicit calculations 
have demonstrated that this is indeed the case. The above "dual gauge-fixing property" holds for the unrenormalized $\mathrm{S}$-matrix. If one imposes the requirement that the renormalized PT $n$-point functions satisfy exactly the same set of WI as their unrenormalized counterparts, one then concludes that the "dual gauge-fixing property" holds also after renormalization. After enforcing this last requirement one finally arrives at a set of conditions relating the various wavefunction- and coupling- renormalization constants of the theory.

To see this in detail, we start by listing the relations between the bare and renormalized parameters for the electroweak sector of the SM. We indicate all (bare) unrenormalized quantities with the superscript ' 0 ', For the masses we have

$$
\begin{gathered}
\left(M_{W}^{0}\right)^{2}=M_{W}^{2}+\delta M_{W}^{2}, \quad\left(M_{Z}^{0}\right)^{2}=M_{Z}^{2}+\delta M_{Z}^{2}, \\
\left(M_{H}^{0}\right)^{2}=M_{H}^{2}+\delta M_{H}^{2}, \quad m_{f}^{0}=m_{f}+\delta m_{f} .
\end{gathered}
$$

In addition, the wave-function renormalizations are given by

$$
\begin{gathered}
W_{\mu}^{ \pm, 0}=\hat{Z}_{W}^{1 / 2} W_{\mu}^{ \pm}, \quad Z_{\mu}^{0}=\hat{Z}_{Z}^{1 / 2} Z_{\mu}, \\
G^{ \pm, 0}=\hat{Z}_{G^{ \pm}}^{1 / 2} G^{ \pm}, \quad G^{0,0}=\hat{Z}_{G^{0}}^{1 / 2} G^{0}, \\
H^{0}=\hat{Z}_{H}^{1 / 2} H, \quad f_{L(R)}^{0}=\hat{Z}_{f}^{L(R) 1 / 2} f_{L(R)}, \\
g_{w}^{0}=\hat{Z}_{g_{w}} g_{w}, \quad c_{w}^{0}=\hat{Z}_{c_{w}} c_{w},
\end{gathered}
$$

with

$$
\hat{Z}_{c_{w}}=\left(1+\frac{\delta M_{W}^{2}}{M_{W}^{2}}\right)^{1 / 2}\left(1+\frac{\delta M_{Z}^{2}}{M_{Z}^{2}}\right)^{-1 / 2} .
$$

If we expand $\hat{Z}_{c_{w}}$ perturbatively, we have

$$
\hat{Z}_{c_{w}}=1+\frac{1}{2} \frac{\delta c_{w}^{2}}{c_{w}^{2}}+\cdots
$$

with

$$
\frac{\delta c_{w}^{2}}{c_{w}^{2}}=\frac{\delta M_{W}^{2}}{M_{W}^{2}}-\frac{\delta M_{Z}^{2}}{M_{Z}^{2}},
$$

which is the usual one-loop result. The carets in the above formulas indicate as usual that both the calculations as well as the renormalization procedure are carried out in the PT framework.

Imposing the requirement that the PT Green's functions should respect the same WI's before and after renormalization we arrive at the following relations:

$$
\begin{aligned}
& \hat{Z}_{W}=\hat{Z}_{g_{w}}^{-2}, \\
& \hat{Z}_{Z}=\hat{Z}_{W} \hat{Z}_{c_{w}}^{2},
\end{aligned}
$$

$$
\begin{aligned}
\hat{Z}_{G^{0}}=\hat{Z}_{G^{ \pm}} & =\hat{Z}_{W}\left(1+\frac{\delta M_{W}^{2}}{M_{W}^{2}}\right), \\
\hat{Z}_{u}^{L} & =\hat{Z}_{d}^{L} .
\end{aligned}
$$

In deriving the above expressions, the following exact algebraic identity may be used

$$
\left(1+\frac{\delta M_{W}^{2}}{M_{W}^{2}}\right)\left(1-\frac{\delta M_{W}^{2}}{\left(M_{W}^{0}\right)^{2}}\right)=1 .
$$

It is important to notice that the relations listed above are exact to all orders in perturbation theory. Instead, in the usual perturbative treatment, one sets $\hat{Z}_{i}^{1 / 2}=1+\frac{1}{2} \delta \hat{Z}_{i}$, for $i$ $=W, Z, H, f$, and neglects higher order terms. For example, at one loop order, i.e., if we neglect terms of order $g^{4}$ and higher, the relation Eq. (5.8) becomes

$$
\delta \hat{Z}_{G^{0}}=\delta \hat{Z}_{G^{ \pm}}=\delta \hat{Z}_{W}+\frac{\delta M_{W}^{2}}{M_{W}^{2}} .
$$

It is instructive to show with an explicit example how the relations in Eq. (5.8) may be derived. To this end, we first define the proper unrenormalized one-loop vertices:

$$
\begin{aligned}
& \bar{\Gamma}_{\mu}^{W^{+} \bar{u} d, 0}\left(q, p_{u}^{-}, p_{d}\right) \equiv \Gamma_{0 \mu}^{W^{+} \bar{u} d, 0}+\hat{\Gamma}_{\mu}^{W^{+} \bar{u} d, 0}\left(q, p_{u}^{-}, p_{d}\right), \\
& \bar{\Gamma}^{G^{+} \bar{u} d, 0}\left(q, p_{u}^{-}, p_{d}\right) \equiv \Gamma_{0}^{G^{+} \bar{u} d, 0}+\hat{\Gamma}^{G^{+} \bar{u} d, 0}\left(q, p_{\bar{u}}^{-}, p_{d}\right),
\end{aligned}
$$

where $\Gamma_{0 \mu}^{W^{+} \bar{u} d, 0}$ and $\hat{\Gamma}_{\mu}^{W^{+} \bar{u} d, 0}$ are the tree-level and one-loop PT $W^{+} \bar{u} d$ vertices, respectively. Correspondingly, $\Gamma_{0}^{G^{+}} \bar{u} d, 0$ and $\hat{\Gamma}^{G^{+}} \bar{u} d, 0$ are the Born-level and one-loop PT $G^{+} \bar{u} d$ vertices. Furthermore, if one neglects quark mixing, the bare dressed $u$ - and $d$-type quark propagators are given by

$$
\begin{aligned}
& \hat{S}_{u}^{0}\left(p_{u}\right)=\left[p_{u}-m_{u}^{0}+\hat{\Sigma}^{\bar{u} u, 0}\left(p_{u}\right)\right]^{-1}, \\
& \hat{S}_{d}^{0}\left(p_{d}\right)=\left[p_{d}-m_{d}^{0}+\hat{\Sigma}^{\bar{d} d, 0}\left(p_{d}\right)\right]^{-1} .
\end{aligned}
$$

Because of the fact that the bare effective PT vertices and self-energies satisfy tree-level WI's, one then has

$$
\begin{aligned}
& q^{\mu} \bar{\Gamma}_{\mu}^{W^{+} \bar{u} d, 0}\left(q, p_{\bar{u}}^{-}, p_{d}\right)+M_{W}^{0} \bar{\Gamma}^{G^{+} \bar{u} d, 0}\left(q, p_{\bar{u}}^{-}, p_{d}\right) \\
& =-\frac{i g_{w}^{0}}{\sqrt{2}}\left[\hat{S}_{u}^{0-1}\left(p_{u}^{-}\right) P_{L}-P_{R} \hat{S}_{d}^{0-1}\left(p_{d}\right)\right] .
\end{aligned}
$$

The renormalized quantities are defined as follows:

$$
\begin{gathered}
\frac{1}{g_{w}} \bar{\Gamma}_{\mu}^{W^{+} \bar{u} d}=\hat{Z}_{W^{+} \bar{u} d} \frac{1}{g_{w}^{0}} \bar{\Gamma}_{\mu}^{W^{+} \bar{u} d, 0}, \\
\frac{1}{g_{w}} \bar{\Gamma}^{G^{+} \bar{u} d}=\hat{Z}_{G^{+} \bar{u} d} \frac{1}{g_{w}^{0}} \bar{\Gamma}^{G^{+} \bar{u} d, 0},
\end{gathered}
$$




$$
\begin{aligned}
& \hat{S}_{d}^{0}\left(p_{d}\right)=\hat{Z}_{d}^{1 / 2} \hat{S}_{d}\left(p_{d}\right) \hat{Z}_{d}^{1 / 2 \dagger}, \\
& \hat{S}_{u}^{0}\left(p_{u}^{-}\right)=\hat{Z}_{u}^{1 / 2} \hat{S}_{u}\left(p_{u}^{-}\right) \hat{Z}_{u}^{1 / 2 \dagger},
\end{aligned}
$$

where $\quad \hat{Z}_{f}^{1 / 2}=\hat{Z}_{f}^{L 1 / 2} P_{L}+\hat{Z}_{f}^{R 1 / 2} P_{R} \quad$ and $\quad \hat{Z}_{G^{+} \bar{u} d}=\hat{Z}_{G^{+} \bar{u} d}^{L} P_{L}$ $+\hat{Z}_{G^{+}{ }^{\prime} d}^{R} P_{R}$. Then, the vertex renormalization constants $\hat{Z}_{W^{+} \bar{u} d}$ and $\hat{Z}_{G^{+}}^{L, R}$ are related to the renormalization constants introduced in Eq. (5.2) as follows:

$$
\begin{aligned}
& \hat{Z}_{W^{+} \bar{u} d}=\hat{Z}_{g_{w}} \hat{Z}_{W}^{1 / 2} \hat{Z}_{d}^{L 1 / 2} \hat{Z}_{u}^{L 1 / 2}, \\
& \hat{Z}_{G^{+}{ }^{-} \bar{d}}^{L}=\hat{Z}_{g_{w}} \hat{Z}_{G^{+}}^{1 / 2} \hat{Z}_{d}^{L 1 / 2} \hat{Z}_{u}^{R 1 / 2}, \\
& \hat{Z}_{G^{+}{ }^{-} d^{R}}^{R}=\hat{Z}_{g_{w}} \hat{Z}_{G^{+}}^{1 / 2} \hat{Z}_{u}^{L 1 / 2} \hat{Z}_{d}^{R 1 / 2} .
\end{aligned}
$$

After replacing the bare by the renormalized quantities in Eq. (5.14) by means of Eqs. (5.16), (5.17), (5.18), and (5.1) for the mass renormalization, we require that the renormalized WI retains its original form, i.e.,

$$
\begin{gathered}
q^{\mu} \bar{\Gamma}_{\mu}^{W^{+} \bar{u} d}\left(q, p_{u}^{-}, p_{d}\right)+M_{W} \bar{\Gamma}^{G^{+} \bar{u} d}\left(q, p_{u}^{-}, p_{d}\right) \\
=-\frac{i g_{w}}{\sqrt{2}}\left[\hat{S}_{u}^{-1}\left(p_{u}^{-}\right) P_{L}-P_{R} \hat{S}_{d}^{-1}\left(p_{d}\right)\right] .
\end{gathered}
$$

The above requirement leads to relations among the renormalization constants within the framework of perturbation theory. From the WI involving the chirality structure $P_{R} \bar{\Gamma}_{\mu}^{W^{+} \bar{u} d, 0} P_{L}$ in Eq. (5.14), we thus obtain that $\hat{Z}_{g_{w}}=\hat{Z}_{W}^{-1 / 2}$ and $\hat{Z}_{u}^{L}=\hat{Z}_{d}^{L}$, which are Eqs. (5.6) and (5.9), respectively. Furthermore, imposing that the form of WI for $P_{L} \bar{\Gamma}_{\mu}^{W^{+} \bar{u} d, 0} P_{L}$ or $P_{R} \bar{\Gamma}_{\mu}^{W^{+} \bar{u} d, 0} P_{R}$ remains the same after renormalization yields $\hat{Z}_{G^{+}}^{1 / 2}=\hat{Z}_{W}^{1 / 2}\left(1+\delta M_{W}^{2} / M_{W}^{2}\right)^{1 / 2}$, which is the last equality of Eq. (5.8).

Following an exactly similar procedure we can derive the rest of the relations listed in Eqs. (5.7) and (5.8).

\section{B. Effective charges for the gauge bosons}

In this sub-section we show how the relations among the renormalization constants derived above enable one to construct a process-independent RGI quantity for the gauge bosons of the theory. For definiteness, we concentrate on the $W$ boson, but similar arguments apply for the photon and the $Z$ boson.

First, we shall show that the bare and renormalized PT resummed $W$-boson propagators, $\Delta_{\mu \nu}^{W, 0}(q)$ and $\hat{\Delta}_{\mu \nu}^{W}(q)$, respectively, satisfy the following relation

$$
\Delta_{\mu \nu}^{W, 0}(q)=\hat{Z}_{W} \hat{\Delta}_{\mu \nu}^{W}(q) .
$$

We start with the most general form of $\hat{\Delta}_{\mu \nu}^{W, 0}(q)$ given by

$$
\hat{\Delta}_{\mu \nu}^{W, 0}(q)=\hat{\Delta}_{T}^{W, 0}\left(q^{2}\right) t_{\mu \nu}+\hat{\Delta}_{L}^{W, 0}\left(q^{2}\right) l_{\mu \nu},
$$

where

$$
t_{\mu \nu}(q)=-g_{\mu \nu}+\frac{q_{\mu} q_{\nu}}{q^{2}}, \quad l_{\mu \nu}(q)=\frac{q_{\mu} q_{\nu}}{q^{2}} .
$$

As was shown in detail in [18], if we decompose the bare one-loop PT $W$-boson self-energy $\hat{\Pi}_{\mu \nu}^{W, 0}(q)$ in the form

$$
\hat{\Pi}_{\mu \nu}^{W, 0}(q)=\hat{\Pi}_{T}^{W, 0}\left(q^{2}\right) t_{\mu \nu}+\hat{\Pi}_{L}^{W, 0}\left(q^{2}\right) l_{\mu \nu},
$$

then in the PT resummation formalism the quantities $\hat{\Delta}_{T}^{W, 0}\left(q^{2}\right)$ and $\hat{\Delta}_{L}^{W, 0}\left(q^{2}\right)$ are given by

$$
\begin{aligned}
& \hat{\Delta}_{T}^{W, 0}\left(q^{2}\right)=\left[q^{2}-\left(M_{W}^{0}\right)^{2}+\hat{\Pi}_{T}^{W, 0}\left(q^{2}\right)\right]^{-1}, \\
& \hat{\Delta}_{L}^{W, 0}\left(q^{2}\right)=\left[\left(M_{W}^{0}\right)^{2}-\hat{\Pi}_{L}^{W, 0}\left(q^{2}\right)\right]^{-1} .
\end{aligned}
$$

The standard renormalization procedure is to define the wave function renormalization, $\hat{Z}_{W}$, by means of the transverse part of the resummed $W$-boson propagator:

$$
\hat{Z}_{W}\left[q^{2}-\left(M_{W}^{0}\right)^{2}+\hat{\Pi}_{T}^{W, 0}\left(q^{2}\right)\right]=q^{2}-M_{W}^{2}+\hat{\Pi}_{T}^{W}\left(q^{2}\right),
$$

where the explicit form of $\hat{Z}_{W}$ depends on the renormalization scheme. Similarly, the propagator of the associated would-be Goldstone boson $G^{+}$is renormalized as usual, i.e.,

$$
\hat{D}^{G^{+}, 0}\left(q^{2}\right)=\left[q^{2}-\hat{\Omega}^{0}\left(q^{2}\right)\right]^{-1}=\hat{Z}_{G^{+}}\left[q^{2}-\hat{\Omega}\left(q^{2}\right)\right]^{-1},
$$

with

$$
G^{ \pm, 0}=\hat{Z}_{G^{+}} G^{ \pm} .
$$

Note that we only need to carry out a wave-function renormalization for the Goldstone boson self-energy $\hat{\Omega}^{0}\left(q^{2}\right)$ (with tadpole and seagull graphs included), since $\hat{\Omega}^{0}(0)=0$, in agreement with the Goldstone theorem, which states that Goldstone bosons are massless to all orders in perturbation theory. The latter is a result of the gauge invariance of the diagrammatic PT method.

From the PT WI's involving the self-energies, we have

$$
\hat{\Pi}_{L}^{W, 0}\left(q^{2}\right)=\frac{\left(M_{W}^{0}\right)^{2}}{q^{2}} \hat{\Omega}^{0}\left(q^{2}\right),
$$

which implies that

$$
\hat{\Delta}_{L}^{W, 0}\left(q^{2}\right)=\frac{q^{2}}{\left(M_{W}^{0}\right)^{2}} \hat{D}^{G^{+}, 0}\left(q^{2}\right) .
$$

This last identity allows us to write the resummed $W$-boson propagator in the form 


$$
\begin{aligned}
\hat{\Delta}_{\mu \nu}^{W, 0}(q)= & \hat{Z}_{W}\left[\hat{\Delta}_{T}^{W}\left(q^{2}\right) t_{\mu \nu}+\hat{Z}_{W}^{-1} \hat{\Delta}_{L}^{W, 0}\left(q^{2}\right) l_{\mu \nu}\right] \\
= & \hat{Z}_{W}\left[\hat{\Delta}_{T}^{W}\left(q^{2}\right) t_{\mu \nu}+\hat{Z}_{W}^{-1} \hat{Z}_{G^{+}}\left(1+\frac{\delta M_{W}^{2}}{M_{W}^{2}}\right)^{-1}\right. \\
& \left.\times \frac{q^{2}}{M_{W}^{2}} \hat{D}^{G^{+}}\left(q^{2}\right) l_{\mu \nu}\right] .
\end{aligned}
$$

Imposing that Eq. (5.28), or equivalently Eq. (5.29), holds for the renormalized quantities as well, we find

$$
\begin{aligned}
\hat{\Delta}_{\mu \nu}^{W, 0}(q)= & \hat{Z}_{W}\left[\hat{\Delta}_{T}^{W}\left(q^{2}\right) t_{\mu \nu}+\hat{Z}_{W}^{-1} \hat{Z}_{G^{+}}\right. \\
& \left.\times\left(1+\frac{\delta M_{W}^{2}}{M_{W}^{2}}\right) \hat{\Delta}_{L}^{W}\left(q^{2}\right) l_{\mu \nu}\right]
\end{aligned}
$$

Finally, using the last equality of Eq. (5.8) we arrive at Eq. (5.20).

It is now straightforward to see that the processindependent RGI quantity for the $W$ boson is given by

$$
\hat{R}_{\mu \nu}^{W, 0}(q)=\frac{\left(g_{w}^{0}\right)^{2}}{4 \pi} \hat{\Delta}_{\mu \nu}^{W, 0}(q)=\frac{\left(g_{w}\right)^{2}}{4 \pi} \hat{\Delta}_{\mu \nu}^{W}(q)=\hat{R}_{\mu \nu}^{W}(q)
$$

At this point one might be tempted to separate the above quantity into the product of a dimension-full kinematic factor and a dimensionless quantity, which could be identified with an effective charge, in direct analogy to the QED and QCD cases. This kind of factorization may however introduce artifacts into both components, which are absent from the original RGI expression. For example, take the simple case where $\hat{R}_{\mu \nu}^{W}$ is sandwiched between conserved external currents (massless external fermions), and let us decompose $\hat{R}_{\mu \nu}^{W}$ in the form

$$
\hat{R}_{\mu \nu}^{W}(q)=\alpha_{w, \mathrm{eff}}\left(q^{2}\right) \frac{-g_{\mu \nu}}{q^{2}-\bar{s}\left(q^{2}\right)}
$$

where $\bar{s}\left(q^{2}\right)$ denotes the position of the physical complex pole of the $W$ boson which appears on the second Riemann sheet. Two possible parametrizations of the pole are $\bar{s}$ $=M_{W}^{2}-i M_{W} \Gamma_{W}$ (constant imaginary part) or $\bar{s}\left(q^{2}\right)=M_{W}^{2}$ $-i q^{2} \Gamma_{W} / M_{W}\left(q^{2}\right.$-dependent imaginary part), where $\Gamma_{W}$ is the constant width of the $W$ boson on the pole. In the first case, we see that at $q^{2}=0$ for example, the kinematic factor is complex, whereas the RGI quantity is real, i.e., Im $\hat{R}_{\mu \nu}^{W}(0)=0$, since, by construction, it only develops an imaginary part at the lowest physical threshold $q^{2}>m_{e}^{2}$. Consequently, $\alpha_{w, \text { eff }}(0)$ is also imaginary, i.e., it contains thresholds which are artifacts of the decomposition. The second parametrization of $\bar{s}\left(q^{2}\right)$ does not have the above problems, but still, the effective charge so defined contains erroneous information about the position of the various decay channels. Similar considerations apply to the case of the $Z$ boson, or other possible gauge bosons appearing in extensions of the SM.

Apart from identifying the process-independent RGI quantity for the $W$ boson $\hat{R}_{\mu \nu}^{W}(q)$ in Eq. (5.32), one can also introduce process-dependent RGI quantities. For instance, to one-loop, the $\gamma W^{-} W^{+}$vertex may be written in the form

$$
\hat{R}_{\mu \nu \lambda}^{\gamma W^{-} W^{+}}\left(q, k_{-}, k_{+}\right)=\left(\frac{4 \pi}{g_{w}^{2}}\right) \frac{1}{e} \bar{\Gamma}_{\mu \nu \lambda}^{\gamma W^{-} W^{+}}\left(q, k_{-}, k_{+}\right),
$$

with

$$
\bar{\Gamma}_{\mu \nu \lambda}^{\gamma W^{-} W^{+}}\left(q, k_{-}, k_{+}\right)=\Gamma_{0 \mu \nu \lambda}^{\gamma W^{-} W^{+}}+\hat{\Gamma}_{\mu \nu \lambda}^{\gamma W^{-} W^{+}}\left(q, k_{-}, k_{+}\right) .
$$

Here, $\Gamma_{0 \mu \nu \lambda}^{\gamma W^{-} W^{+}}$and $\hat{\Gamma}_{\mu \nu \lambda}^{\gamma W^{-} W^{+}}\left(q, k_{-}, k_{+}\right)$are the tree-level and one-loop PT $\gamma W^{-} W^{+}$couplings, respectively. The quantity $\hat{R}_{\mu \nu \lambda}^{\gamma W^{-} W^{+}}\left(q, k_{-}, k_{+}\right)$is UV finite and invariant under the renormalization group. This can easily be shown by means of the one-loop PT WI, which can also be written in a RGI form, viz.,

$$
q^{\mu} \hat{R}_{\mu \nu \lambda}^{\gamma W^{-} W^{+}}\left(q, k_{-}, k_{+}\right)=\hat{R}_{\nu \lambda}^{W-1}\left(k_{-}\right)-\hat{R}_{\nu \lambda}^{W-1}\left(k_{+}\right) .
$$

This last equation shows how the action of $q^{\mu}$ on $\hat{R}_{\mu \nu \lambda}^{\gamma W^{-} W^{+}}$ projects out the process-independent part of the $\gamma W^{-} W^{+}$ vertex, which is related to the $W$-boson effective charge. Similarly, one can construct RGI combinations for all the PT vertices related to couplings, e.g., $\gamma W^{-} G^{+}, Z W^{-} W^{+}$, $Z G^{-} G^{+}$, etc. In particular, at LEP2, it is very advantageous to use the RGI expressions $\hat{R}_{\mu \nu \lambda}^{\gamma W^{-} W^{+}}$and $\hat{R}_{\mu \nu \lambda}^{Z W^{-} W^{+}}$, which lead to UV finite form-factors for the $\gamma W^{-} W^{+}$and $Z W^{-} W^{+}$ vertices.

\section{The effective charge of the Higgs boson}

We now proceed to extend the notion of the effective charge to the case of the Higgs boson. For this purpose, we first express the unrenormalized Higgs-boson propagator in terms of the renormalized one as follows:

$$
\begin{aligned}
\hat{\Delta}^{H, 0}\left(q^{2}\right) & =\left[q^{2}-\left(M_{H}^{0}\right)^{2}+\hat{\Pi}^{H H, 0}\left(q^{2}\right)\right]^{-1} \\
& =\hat{Z}_{H}\left[q^{2}-M_{H}^{2}+\hat{\Pi}^{H H}\left(q^{2}\right)\right]^{-1}=\hat{Z}_{H} \hat{\Delta}^{H}\left(q^{2}\right),
\end{aligned}
$$

where $M_{H}$ may be defined to be the real part of the complex pole position of $\hat{\Delta}^{H}\left(q^{2}\right)$. Following a procedure rather similar to that given in Sec. V A, we should exploit the gauge symmetry of the SM, in order to deduce relations between $\hat{Z}_{H}$ and the other renormalization constants.

We start again with the PT WI relating the one-loop vertices $H Z G^{0}$ and $H G^{0} G^{0}$, i.e., 


$$
\begin{array}{r}
k_{1}^{\mu} \bar{\Gamma}_{\mu}^{H Z G^{0}, 0}\left(q, k_{1}, k_{2}\right)+i M_{Z}^{0} \bar{\Gamma}^{H G^{0} G^{0}, 0}\left(q, k_{1}, k_{2}\right) \\
=-\frac{g_{w}^{0}}{2 c_{w}^{0}}\left[\left(\hat{\Delta}^{H, 0}\left(q^{2}\right)\right)^{-1}-\left(\hat{D}^{G^{0}, 0}\left(k_{2}^{2}\right)\right)^{-1}\right],
\end{array}
$$

where

$$
\begin{aligned}
\hat{D}^{G^{0}, 0}\left(k^{2}\right) & =\left[k^{2}-\hat{\Pi}^{G^{0} G^{0}, 0}\left(k^{2}\right)\right]^{-1} \\
& =\hat{Z}_{G^{0}}\left[k^{2}-\hat{\Pi}^{G^{0} G^{0}}\left(k^{2}\right)\right]^{-1}
\end{aligned}
$$

and

$$
\begin{gathered}
\bar{\Gamma}_{\mu}^{H Z G^{0}, 0} \equiv \Gamma_{0 \mu}^{H Z G^{0}, 0}+\hat{\Gamma}_{\mu}^{H Z G^{0}, 0}, \\
\bar{\Gamma}^{H G^{0} G^{0}, 0} \equiv \Gamma_{0}^{H G^{0} G^{0}, 0}+\hat{\Gamma}^{H G^{0} G^{0}, 0} .
\end{gathered}
$$

As before, in Eq. (5.39), the subscript " 0 "' denotes tree level couplings, while the caret indicates one-loop vertices obtained in the PT.

As usual, we write the unrenormalized $H Z G^{0}$ and $H G^{0} G^{0}$ vertices as a product of the renormalized ones and a vertex renormalization constant,

$$
\begin{gathered}
\frac{2 c_{w}}{g_{w}} \bar{\Gamma}_{\mu}^{H Z G^{0}}=\hat{Z}_{H Z G^{0}} \frac{2 c_{w}^{0}}{g_{w}^{0}} \bar{\Gamma}_{\mu}^{H Z G^{0}, 0}, \\
\frac{2 c_{w}}{g_{w}} \bar{\Gamma}^{H G^{0} G^{0}}=\hat{Z}_{H G^{0} G^{0}} \frac{2 c_{w}^{0}}{g_{w}^{0}} \bar{\Gamma}^{H G^{0} G^{0}, 0} .
\end{gathered}
$$

Making use of the fact that the effective $H Z G^{0}$ and $H G^{0} G^{0}$ vertices are completely renormalized by a redefinition of the fields and the couplings given in Eq. (5.2), we find

$$
\begin{aligned}
& \hat{Z}_{H Z G^{0}}=\hat{Z}_{g_{w}} \hat{Z}_{c_{w}}^{-1} \hat{Z}_{Z}^{1 / 2} \hat{Z}_{H}^{1 / 2} \hat{Z}_{G^{0}}^{1 / 2}=\hat{Z}_{H}^{1 / 2} \hat{Z}_{G^{0}}^{1 / 2}, \\
& \hat{Z}_{H G^{0} G^{0}}=\hat{Z}_{g_{w}} \hat{Z}_{c_{w}}^{-1} \hat{Z}_{H}^{1 / 2} \hat{Z}_{G^{0}}=\hat{Z}_{H}^{1 / 2} \hat{Z}_{G^{0}}^{1 / 2}\left(1+\frac{\delta M_{Z}^{2}}{M_{Z}^{2}}\right)^{1 / 2} .
\end{aligned}
$$

In the last step of Eqs. (5.41) and (5.42), we have used the relations given in Eqs. (5.6)-(5.8).

In order that the PT WI in Eq. (5.38) maintains the same form after renormalization, it is necessary to have

$$
\hat{Z}_{H}=\hat{Z}_{G^{0}}
$$

Employing Eqs. (5.6), (5.8), and (5.43), it is easy to show that

$$
\begin{aligned}
\hat{R}^{H, 0}\left(q^{2}\right) & =\frac{\left(g_{w}^{0}\right)^{2}}{\left(M_{W}^{0}\right)^{2}} \hat{\Delta}^{H, 0}\left(q^{2}\right) \\
& =\left[\frac{g_{w}^{2}}{M_{W}^{2}} \hat{\Delta}^{H}\left(q^{2}\right)\right] \hat{Z}_{g}^{2} \hat{Z}_{H}\left(1+\frac{\delta M_{W}^{2}}{M_{W}^{2}}\right)^{-1}=\hat{R}^{H}\left(q^{2}\right)
\end{aligned}
$$

is a process-independent RGI quantity, in close analogy to the RGI quantity of the $W$ boson $\hat{R}_{\mu \nu}^{W}(q)$. As a byproduct of this, we also find that $\hat{R}^{G^{+}}\left(k^{2}\right)=\left(g_{w}^{2} / M_{W}^{2}\right) \hat{D}^{G^{+}}\left(k^{2}\right)$ and $\hat{R}^{G^{0}}\left(k^{2}\right)=\left(g_{w}^{2} / M_{W}^{2}\right) \hat{D}^{G^{0}}\left(k^{2}\right)$ are invariant under the renormalization group. Hence, we conclude that the quantity $\hat{R}^{H}\left(q^{2}\right)$ provides a natural generalization of the concept of the effective charge in the case of the Higgs boson. It is interesting to notice the exact analogy between the form of the Higgs boson effective charge of Eq. (5.44) obtained within a theory with a non-Abelian gauge symmetry such as the SM, and that of Eq. (4.14) derived in the context of a much simpler model with Abelian global (un-gauged) symmetry.

Finally, a direct derivation of the above general result may be obtained if one adopts the symmetric formulation of the classical action in the BFM [40]. Within this formulation one is led to the minimal on-shell scheme, with the relevant renormalization constants satisfying

$$
\hat{\Phi}^{0}=Z_{\hat{\Phi}}^{1 / 2} \hat{\Phi}, \quad \hat{v}^{0}=Z_{\hat{\Phi}}^{1 / 2}(\hat{v}+\delta \hat{v}) .
$$

Using the above relations, and the additional fact that, due to the background symmetry, in this formulation $\delta \hat{v}=0$, one can immediately show that

$$
\left(\hat{v}^{0}\right)^{-2}\left\langle 0\left|T: \hat{\Phi}^{0}(x) \hat{\Phi}^{0}(y):\right| 0\right\rangle=\hat{v}^{-2}\langle 0|T: \hat{\Phi}(x) \hat{\Phi}(y):| 0\rangle,
$$

which is Eq. (5.44). Even though the analysis of this subsection which led to Eq. (5.44) is more general since it does not rely on any particular gauge-fixing procedure or renormalization scheme, this latter derivation within the symmetric BFM framework has the advantage of directly generalizing the construction of the scalar effective charge given for the toy model of Sec. IV C to the realistic case of the SM.

\section{DIAGRAMMATIC ANALYSIS OF THE EQUIVALENCE THEOREM}

Cornwall, Levin and Tiktopoulos, and shortly afterwards Vayonakis, showed that at very high energies the amplitude for emission or absorption of a longitudinally polarized gauge boson becomes equal to the amplitude in which the gauge boson is replaced by the corresponding would-be Goldstone boson [13]. The above statement is a consequence of the underlying local gauge invariance of the SM, and is known as the equivalence theorem (ET); it has been proven to hold to all orders in perturbation theory for multiple absorptions and emissions of massive vector bosons [14]. Compliance with this theorem is a necessary requirement for any resummation algorithm, since any Born-improved amplitude 
which fails to satisfy it is bound to be missing important physical information. The reason why most resummation methods are at odds with the ET is that in the usual diagrammatic analysis the underlying symmetry of the amplitudes is not manifest; just as happens in the case of the OT, the conventional sub-amplitudes defined in terms of Feynman diagrams do not satisfy the ET individually. The resummation of such a sub-amplitude will in turn distort several subtle cancellations, thus giving rise to artifacts and unphysical effects. Instead, as we will show in detail in this section, the PT sub-amplitudes satisfy the ET individually. As is common in the PT framework, the only non-trivial step for accomplishing this is the proper exploitation of elementary WI's. In addition, the part of the Born-improved amplitude containing the resummed Higgs boson self-energy (or the RGI quantity defined in Sec. V C) satisfies the ET independently of the rest of the (non-resummed) amplitude. This is explicitly demonstrated by resorting almost exclusively to the fact that, in contrast to their conventional counterparts, the one-loop Higgs-boson vertices defined within the PT satisfy naive, tree-level WI's.

The formal derivation of the ET is based on the observation [14] that, by virtue of the Becchi-Rouet-Stora (BRS) invariance of the theory, the connected transition amplitude between physical states of any number $n$ of insertions of the gauge-fixing term $F^{a}$ vanishes, i.e.,

$$
\left\langle f\left|T: F^{a_{1}}\left(x_{1}\right) F^{a_{2}}\left(x_{2}\right) \ldots F^{a_{n}}\left(x_{n}\right):\right| i\right\rangle_{\text {con. }}=0 .
$$

In the renormalizable $R_{\xi}$ gauges, $F^{a}$ assumes the form

$$
F^{a}(x)=\partial^{\mu} V_{\mu}^{a}(x)+\xi_{a} M_{V^{a}} G^{a}(x),
$$

where $V_{\mu}^{a}$ denotes the massive gauge boson, e.g., $W^{ \pm}$or $Z$, $G^{a}$ its corresponding would-be Goldstone boson, e.g., $G^{ \pm}$or $G^{0}, \xi_{a}$ its GFP, and $M_{V^{a}}$ its mass. Since for energies $E_{V}$ $\gg M_{V}$ the longitudinal polarization vector $\varepsilon_{L}^{\mu}(k)$ of the gauge boson $V$ behaves as

$$
\varepsilon_{L}^{\mu}(k)=\frac{k^{\mu}}{M_{V}}+v^{\mu}(k),
$$

with $v_{\mu}(k)=\mathcal{O}\left(M_{V} / E_{V}\right)$, in the configuration space $\varepsilon_{L}^{\mu}(k)$ may be represented naively by the derivative $\partial_{\mu} / M_{V}$, which in turn enables one to use the identities derived from Eq. (6.1). Beyond the tree level, one has in general to include correction factors [41], denoted here as $K^{a_{i}}$, which take into account renormalization effects. Finally, given that, due to the unitarity of the SM, amplitudes involving only would-be Goldstone bosons cannot grow faster than a constant at high energies, one finally arrives at

$$
\mathcal{T}\left(V_{L}^{a_{1}} \ldots V_{L}^{a_{n}} ; X\right)=\prod_{i=1}^{n} K^{a_{i}} \mathcal{T}\left(G^{a_{1}} \ldots G^{a_{n}} ; X\right)+\mathcal{O}(M / E),
$$

where $V_{L}^{\alpha} \equiv \varepsilon_{L}^{\mu} V_{\mu}^{\alpha}$, and $X$ denotes all other fields. The above equality represents the ET in its most basic form. Note that the ET cannot give any interesting information for ampli-

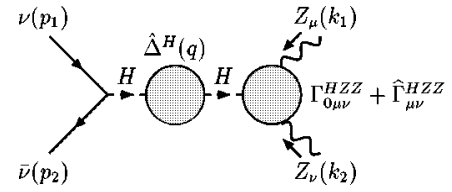

(a)

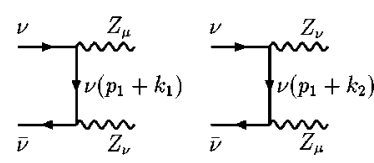

(b) (c)
FIG. 6. Resummation of the Higgs-mediated amplitude pertinent to $\nu \bar{\nu} \rightarrow Z Z$.

tudes which decrease in magnitude as $1 / \sqrt{s}$ or faster with increasing energy $E \approx \sqrt{s}$. In order to obtain non-trivial information for the energetically suppressed terms of order $M / \sqrt{s}$ and their higher powers, one has to invoke the socalled generalized ET (GET) [14], whose derivation again relies on the identities of Eq. (6.1). In the case of amplitudes with two longitudinal $W^{+}$bosons for example, the GET establishes the following relation:

$$
\begin{aligned}
& \mathcal{T}\left[W_{L}^{+, \mu}\left(k_{+}\right) W_{L}^{-, \nu}\left(k_{-}\right) ; X\right] \\
&= K^{+} K^{-} \mathcal{T}\left[G^{+}\left(k_{+}\right) G^{-}\left(k_{-}\right) ; X\right] \\
&+K^{+} \mathcal{T}\left[G^{+}\left(k_{+}\right) w^{-, \nu}\left(k_{-}\right) ; X\right] \\
&+K^{-} \mathcal{T}\left[w^{+, \mu}\left(k_{+}\right) G^{-}\left(k_{-}\right) ; X\right] \\
&+\mathcal{T}\left[w^{+, \mu}\left(k_{+}\right) w^{-, \nu}\left(k_{-}\right) ; X\right],
\end{aligned}
$$

and for two longitudinal $Z$ bosons:

$$
\begin{aligned}
\mathcal{T}\left[Z_{L}^{\mu}\left(k_{1}\right) Z_{L}^{\nu}\left(k_{2}\right) ; X\right]= & \left(K^{0}\right)^{2} \mathcal{T}\left[G^{0}\left(k_{1}\right) G^{0}\left(k_{2}\right) ; X\right] \\
& +K^{0} \mathcal{T}\left[G^{0}\left(k_{1}\right) z^{\nu}\left(k_{2}\right) ; X\right] \\
& +K^{0} \mathcal{T}\left[z^{\mu}\left(k_{1}\right) G^{0}\left(k_{2}\right) ; X\right] \\
& +\mathcal{T}\left[z^{\mu}\left(k_{1}\right) z^{\nu}\left(k_{2}\right) ; X\right]
\end{aligned}
$$

where $w^{ \pm, \mu}\left(k_{ \pm}\right)=\varepsilon_{L}^{\mu}\left(k_{ \pm}\right)-k_{ \pm}^{\mu} / M_{Z}$ and $z^{\mu}\left(k_{1,2}\right)=\varepsilon_{L}^{\mu}\left(k_{1,2}\right)$ $-k_{1,2}^{\mu} / M_{W}$ are the energetically suppressed parts of the longitudinally polarized $W^{ \pm}$and $Z$ bosons, respectively. In addition, $K^{ \pm}$and $K^{0}$ are renormalization correction factors mentioned above. In the Born approximation, they take the values $K^{+}=-1, K^{-}=1$, and $K^{0}=-i$, if the four-momenta of the gauge bosons are incoming [42], and reverse their sign in the opposite case. Formulas analogous to Eqs. (6.5) and (6.6) can be derived for an arbitrary number of longitudinally polarized $W$ and $Z$ bosons. In the following, we will restrict ourselves to amplitudes involving two vector bosons only.

Let us consider the process $\nu\left(p_{1}\right) \bar{\nu}\left(p_{2}\right) \rightarrow Z_{L}^{\mu}\left(k_{1}\right) Z_{L}^{\nu}\left(k_{2}\right)$ at the tree level, where $\nu$ is a Dirac neutrino with mass $m$ and the four-momenta of the $Z$ boson are defined to enter the interaction vertices as shown in Fig. 6. The total matrix element $\mathcal{T}\left(\nu \bar{\nu} \rightarrow Z_{L} Z_{L}\right)$ is the sum of two amplitudes:

$$
\mathcal{T}\left(\nu \bar{\nu} \rightarrow Z_{L} Z_{L}\right)=\mathcal{T}_{s}^{H}\left(Z_{L} Z_{L}\right)+\mathcal{T}_{t}\left(Z_{L} Z_{L}\right),
$$

where

$$
\mathcal{T}_{s}^{H}\left(Z_{L} Z_{L}\right)=\varepsilon_{L}^{\mu}\left(k_{1}\right) \varepsilon_{L}^{\nu}\left(k_{2}\right) \mathcal{T}_{s \mu \nu}^{H}(Z Z),
$$




$$
\mathcal{T}_{t}\left(Z_{L} Z_{L}\right)=\varepsilon_{L}^{\mu}\left(k_{1}\right) \varepsilon_{L}^{\nu}\left(k_{2}\right) \mathcal{T}_{t \mu \nu}(Z Z)
$$

with

$$
\begin{aligned}
\mathcal{T}_{s \mu \nu}^{H}(Z Z)= & -i \Gamma_{0 \mu \nu}^{H Z Z} \Delta_{H}\left(k_{1}+k_{2}\right)\left(\frac{g_{w} m}{2 M_{W}}\right) \bar{v}\left(p_{2}\right) u\left(p_{1}\right), \\
\mathcal{T}_{t \mu \nu}(Z Z)= & -\left(\frac{g_{w}}{2 c_{w}}\right)^{2} \bar{v}\left(p_{2}\right)\left(\gamma_{\nu} P_{L} \frac{1}{p_{1}+k_{1}-m} \gamma_{\mu} P_{L}\right. \\
& \left.+\gamma_{\mu} P_{L} \frac{1}{p_{1}+k_{2}-m} \gamma_{\nu} P_{L}\right) u\left(p_{1}\right) .
\end{aligned}
$$

In Eq. (6.10), the tree-level $H Z Z$ coupling is defined as $\Gamma_{0 \mu \nu}^{H Z Z}=i g_{w} M_{Z}^{2} / M_{W} g_{\mu \nu}$. The entire amplitude $\mathcal{T}(\nu \bar{\nu}$ $\rightarrow Z_{L} Z_{L}$ ) satisfies of course the GET (and hence the ET). What we wish to investigate here is whether the GET holds for the Higgs-mediated part of the amplitude independently. The reason we turn directly to the GET instead of the ET, is simply that both the $\mathcal{T}_{s}^{H}\left(Z_{L} Z_{L}\right)$ of Eq. (6.10) and the amplitude $\mathcal{T}_{s}^{H}\left(G^{0} G^{0}\right)$ given by

$$
\mathcal{T}_{s}^{H}\left(G^{0} G^{0}\right)=-i \Gamma_{0}^{H G^{0} G^{0}} \Delta_{H}\left(k_{1}+k_{2}\right)\left(\frac{g_{w} m}{2 M_{W}}\right) \bar{v}\left(p_{2}\right) u\left(p_{1}\right)
$$

where $\Gamma_{0}^{H G^{0} G^{0}}=-i g_{w} M_{H}^{2} /\left(2 M_{W}\right)$, decrease at very high energies as $1 / s$, because of the presence of the Higgs-boson propagator in the $s$ channel. So, the ET in this case will only furnish trivial information. Instead, according to the GET [14], additional amplitudes should be taken into account if one wishes to keep track of energetically suppressed terms to order $M_{Z}^{2} / s$.

In order to address the question raised above, we will calculate the LHS of Eq. (6.6) explicitly, using full expressions for the longitudinal polarization vectors involved, and check whether the result so obtained coincides or not with the sum of the Higgs-boson-dependent parts of the amplitudes appearing on the RHS of Eq. (6.6) (with $X=\nu \bar{\nu}$ ). For that purpose, we first write the longitudinal polarization vector of the gauge boson $V$ in the covariant form

$$
\varepsilon_{L}^{\mu}(k)=\frac{1}{2 \beta M_{V}}\left[\left(1+\beta^{2}\right) k^{\mu}-\left(1-\beta^{2}\right) \tilde{k}^{\mu}\right],
$$

where $k^{\mu}=\left(E_{V}, \vec{k}_{V}\right)$ is the four-momentum of the $V$ boson, $\widetilde{k}^{\mu}=k_{\mu}$ and $\beta=\left|\vec{k}_{V}\right| / E_{V}$ is the $V$-boson velocity. It is convenient to work in the c.m. system of the process $\nu \bar{\nu}$ $\rightarrow Z_{L}^{\mu}\left(k_{1}\right) Z_{L}^{\nu}\left(k_{2}\right)$; in that case the polarization vector $\varepsilon_{L}^{\mu}\left(k_{1}\right)$ of the $Z^{\mu}$ boson can be expressed in terms of the fourmomenta $k_{1}^{\mu}$ and $\widetilde{k}_{1}^{\mu}=k_{2}^{\mu}$, and $\beta=\left(1-4 M_{Z}^{2} / s\right)^{1 / 2}$. Likewise, $\varepsilon_{L}^{\nu}\left(k_{2}\right)$ is written in terms of $k_{2}^{\nu}$ and $\widetilde{k}_{2}^{\nu}=k_{1}^{\nu}$. To order $M_{Z}^{4} / s^{2}$, the energetically subleading part $z^{\mu}\left(k_{1}\right)$ of $\varepsilon_{L}^{\mu}\left(k_{1}\right)$ is obtained by

$$
z^{\mu}\left(k_{1}\right)=\varepsilon_{L}^{\mu}\left(k_{1}\right)-\frac{k_{1}^{\mu}}{M_{Z}}=-\frac{2 M_{Z}}{s} k_{2}^{\mu}+\mathcal{O}\left(\frac{M_{Z}^{4}}{s^{2}}\right) .
$$

Furthermore, the residual vector $z^{\mu}\left(k_{1}\right)$ has the following properties:

$$
z_{\mu}\left(k_{1}\right) k_{1}^{\mu}=-M_{Z}, \quad z_{\mu}\left(k_{1}\right) z^{\mu}\left(k_{1}\right)=0 .
$$

Exactly analogous formulas and relations hold for $\varepsilon_{L}^{\nu}\left(k_{2}\right)$. Using the decomposition (6.3) for $\varepsilon_{L}^{\mu, \nu}\left(k_{1,2}\right)$ and the properties (6.15) for $z^{\mu, \nu}\left(k_{1,2}\right)$, we find for the part of the amplitude depending on the Higgs boson

$$
\mathcal{T}_{s}^{H}\left(Z_{L} Z_{L}\right)=-\mathcal{T}_{s}^{H}\left(G^{0} G^{0}\right)+\Delta \mathcal{T}_{s}^{H}+\mathcal{T}_{s}^{H}(z z)-\mathcal{T}_{P}^{H}\left(Z_{L} Z_{L}\right),
$$

where $\mathcal{T}_{s}^{H}(z z)=z^{\mu} z^{\nu} \mathcal{T}_{s \mu \nu}^{H}(Z Z)$ and

$$
\Delta \mathcal{T}_{s}^{H}=-\left(\frac{g_{w} m}{2 M_{W}}\right)\left(\frac{g_{w} M_{Z}^{2}}{M_{W}}\right) \Delta_{H}\left(k_{1}+k_{2}\right) \bar{v}\left(p_{2}\right) u\left(p_{1}\right),
$$

and $\mathcal{T}_{P}^{H}\left(Z_{L} Z_{L}\right)$ is the expression given in Eq. (3.11), with $m_{t}$ replaced by $m$. It is now straightforward to verify that

$$
\Delta \mathcal{T}_{s}^{H}=-i \mathcal{T}_{s}^{H}\left(z G^{0}\right)-i \mathcal{T}_{s}^{H}\left(G^{0} z\right),
$$

with

$$
\begin{aligned}
\mathcal{T}_{s}^{H}\left(z G^{0}\right)+\mathcal{T}_{s}^{H}\left(G^{0} z\right) \\
=z^{\mu}\left(k_{1}\right) \mathcal{T}_{s \mu}^{H}\left(Z G^{0}\right)+z^{\nu}\left(k_{2}\right) \mathcal{T}_{s \nu}^{H}\left(G^{0} Z\right) \\
=-i\left[z^{\mu}\left(k_{1}\right) \Gamma_{0 \mu}^{H Z G^{0}}+z^{\nu}\left(k_{2}\right) \Gamma_{0 \nu}^{H G^{0} Z}\right] \\
\quad \times\left(\frac{g_{w} m}{2 M_{W}}\right) \Delta_{H}\left(k_{1}+k_{2}\right) \bar{v}\left(p_{2}\right) u\left(p_{1}\right) .
\end{aligned}
$$

The tree-level $H(q) Z_{\mu}\left(k_{1}\right) G^{0}\left(k_{2}\right)$ coupling in Eq. (6.19) is given by $\Gamma_{0 \mu}^{H Z G^{0}}=g_{w}\left(q-k_{2}\right)_{\mu} /\left(2 c_{w}\right)$, with all momenta defined as incoming. Clearly, the first three terms on the RHS of Eq. (6.16) are nothing but the sum of the Higgs-bosondependent parts of the amplitudes

$$
\begin{gathered}
\mathcal{T}\left[G^{0}\left(k_{1}\right) G^{0}\left(k_{2}\right) ; \nu \bar{\nu}\right], \\
\mathcal{T}\left[z\left(k_{1}\right) G^{0}\left(k_{2}\right) ; \nu \bar{\nu}\right]+\mathcal{T}\left[G^{0}\left(k_{1}\right) z\left(k_{2}\right) ; \nu \bar{\nu}\right]
\end{gathered}
$$

and

$$
\mathcal{T}\left[z\left(k_{1}\right) z\left(k_{2}\right) ; \nu \bar{\nu}\right]
$$

Evidently, the only reason preventing $\mathcal{T}_{s}^{H}\left(Z_{L} Z_{L}\right)$ from satisfying individually the GET is the presence of the term $\mathcal{T}_{P}^{H}\left(Z_{L} Z_{L}\right)$ on the RHS of Eq. (6.16).

However, according to the PT, the genuine Higgs-bosondependent part of the amplitude, $\hat{\mathcal{T}}_{s}^{H}\left(Z_{L} Z_{L}\right)$, is obtained after recognizing that the momenta $k_{1}^{\mu}$ and $k_{2}^{\nu}$ coming from the polarization vectors of the longitudinal $Z$ bosons can extract 
a $s$-channel-like, Higgs-boson-dependent part from the nonresonant amplitude $\mathcal{T}_{t \mu \nu}$, in exactly the same way as happens in Eq. (3.11), namely

$$
\frac{k_{1}^{\mu}}{M_{Z}} \frac{k_{2}^{\nu}}{M_{Z}} \mathcal{T}_{t \mu \nu}(Z Z)=\mathcal{T}_{P}^{H}\left(Z_{L} Z_{L}\right)+\cdots
$$

where, as in Eq. (3.11), the ellipses denote additional contributions not related to the Higgs boson. Thus, $\hat{\mathcal{T}}_{s}^{H}\left(Z_{L} Z_{L}\right)$ $=\mathcal{T}_{s}^{H}\left(Z_{L} Z_{L}\right)+\mathcal{T}_{P}^{H}\left(Z_{L} Z_{L}\right)$.

We now want to check if $\hat{\mathcal{T}}_{s}^{H}\left(Z_{L} Z_{L}\right)$ satisfies the GET; for that to happen one must show that

$$
\begin{aligned}
\hat{\mathcal{T}}_{s}^{H}\left(Z_{L} Z_{L}\right)= & \hat{\mathcal{T}}_{s}^{H}\left(G^{0} G^{0}\right)+\hat{\mathcal{T}}_{s}^{H}\left(G^{0} z\right)+\hat{\mathcal{T}}_{s}^{H}\left(z G^{0}\right)+\hat{\mathcal{T}}_{s}^{H}(z z) \\
= & -\mathcal{T}_{s}^{H}\left(G^{0} G^{0}\right)-i \mathcal{T}_{s}^{H}\left(z G^{0}\right)-i \mathcal{T}_{s}^{H}\left(G^{0} z\right) \\
& +\mathcal{T}_{s}^{H}(z z)-\mathcal{T}_{P}^{H}\left(G^{0} G^{0}\right)-i \mathcal{T}_{P}^{H}\left(G^{0} z\right) \\
& -i \mathcal{T}_{P}^{H}\left(z G^{0}\right)+\mathcal{T}_{P}^{H}(z z)
\end{aligned}
$$

where the amplitudes $\mathcal{T}_{P}^{H}\left(G^{0} G^{0}\right), \mathcal{T}_{P}^{H}\left(G^{0} z\right), \mathcal{T}_{P}^{H}\left(z G^{0}\right)$, and $\mathcal{T}_{P}^{H}(z z)$ denote possible Higgs-boson-dependent $s$-channel pinch parts coming from the $t$-channel amplitudes $\mathcal{T}_{t}\left(G^{0} G^{0}\right)$, $\mathcal{T}_{t}\left(G^{0} z\right), \mathcal{T}_{t}\left(z G^{0}\right)$, and $\mathcal{T}_{t}(z z)$, respectively. It is easy to convince oneself however that $\mathcal{T}_{P}^{H}\left(G^{0} G^{0}\right)=\mathcal{T}_{P}^{H}\left(G^{0} z\right)$ $=\mathcal{T}_{P}^{H}\left(z G^{0}\right)=\mathcal{T}_{P}^{H}(z z)=0$; this is so because, according to Eq. (6.14), the energetically subleading parts $z^{\mu}\left(k_{1}\right)$ and $z^{\nu}\left(k_{2}\right)$ are proportional to the "wrong" momenta, i.e. $k_{2}^{\mu}$ and $k_{1}^{\nu}$, respectively, instead of $k_{1}^{\mu}$ and $k_{2}^{\nu}$, which are necessary for pinching. Therefore, Eq. (6.21) reduces to

$$
\begin{aligned}
\hat{\mathcal{T}}_{s}^{H}\left(Z_{L} Z_{L}\right)= & -\mathcal{T}_{s}^{H}\left(G^{0} G^{0}\right)-i \mathcal{T}_{s}^{H}\left(z G^{0}\right) \\
& -i \mathcal{T}_{s}^{H}\left(G^{0} z\right)+\mathcal{T}_{s}^{H}(z z) .
\end{aligned}
$$

But this last equation is immediately true, by virtue of Eq. (6.16) and the definition of $\hat{\mathcal{T}}_{s}^{H}\left(Z_{L} Z_{L}\right)$.

It is important to stress that Eq. (6.22) demonstrates explicitly how the tree-level Higgs-mediated part of the amplitude $\mathcal{T}_{s}^{H}$ satisfies the GET independently, provided the pinching contribution $\mathcal{T}_{P}^{H}$ residing in the non-resonant amplitude is taken properly into consideration. This fact reveals an underlying relation between the PT and the ET at the diagrammatic level, and constitutes a major result of this paper.

We will now show that within the PT framework the equality (6.22) remains valid even after the Higgs-boson propagator has been resummed. As explained in $[18,19]$, the effective one-loop PT $H Z Z$ vertex $\hat{\Gamma}_{\mu \nu}^{H Z Z}\left(q, k_{1}, k_{2}\right)$ must be included in the amplitude containing the resummed Higgs boson propagator $\hat{\Delta}^{H}(q)$; this is so, because the one-loop PT $H(q) Z^{\mu}\left(k_{1}\right) Z^{\nu}\left(k_{2}\right)$ vertex satisfies a number of tree-level WI's which are crucial for ensuring the gauge invariance of the resummed Higgs-mediated part of the amplitude

$$
\begin{aligned}
\overline{\mathcal{T}}_{s \mu \nu}^{H}\left(Z_{L} Z_{L}\right)= & -i\left[\Gamma_{0 \mu \nu}^{H Z Z}+\hat{\Gamma}_{\mu \nu}^{H Z Z}\left(q, k_{1}, k_{2}\right)\right] \\
& \times\left(\frac{g_{w} m}{2 M_{W}}\right) \hat{\Delta}^{H}(q) \bar{v}\left(p_{2}\right) u\left(p_{1}\right) .
\end{aligned}
$$

The PT WI's identities are

$$
\begin{gathered}
k_{2}^{\nu} \hat{\Gamma}_{\mu \nu}^{H Z Z}\left(q, k_{1}, k_{2}\right)+i M_{Z} \hat{\Gamma}_{\mu}^{H Z G^{0}}\left(q, k_{1}, k_{2}\right) \\
=-\frac{g_{w}}{2 c_{w}} \hat{\Pi}_{\mu}^{Z G^{0}}\left(k_{1}\right), \\
k_{1}^{\mu} \hat{\Gamma}_{\mu}^{H Z G^{0}}\left(q, k_{1}, k_{2}\right)+i M_{Z} \hat{\Gamma}^{H G^{0} G^{0}}\left(q, k_{1}, k_{2}\right) \\
=-\frac{g_{w}}{2 c_{w}}\left[\hat{\Pi}^{H H}\left(q^{2}\right)+\hat{\Pi}^{G^{0} G^{0}}\left(k_{2}^{2}\right)\right] \\
k_{1}^{\mu} k_{2}^{\nu} \hat{\Gamma}_{\mu \nu}^{H Z Z}\left(q, k_{1}, k_{2}\right)+M_{Z}^{2} \hat{\Gamma}^{H G^{0} G^{0}}\left(q, k_{1}, k_{2}\right) \\
=\frac{i g_{w} M_{Z}}{2 c_{w}}\left[\hat{\Pi}^{H H}\left(q^{2}\right)+\hat{\Pi}^{G^{0} G^{0}}\left(k_{1}^{2}\right)+\hat{\Pi}^{G^{0} G^{0}}\left(k_{2}^{2}\right)\right] .
\end{gathered}
$$

As before, we define all momenta to flow into the $H Z Z$ vertex with $q+k_{1}+k_{2}=0$. The closed form of the effective one-loop PT HZZ coupling is given in Appendix B. Note that exactly the same WI's hold true for the tree-level $H Z Z$ coupling before quantizing the classical action by introducing gauge-fixing terms and ghost fields. To be specific, the tree-level WI's derived from the classical action are recovered from Eqs. (6.24)-(6.26) if $\hat{\Pi}^{H H}\left(q^{2}\right)$ and $-\hat{\Pi}^{G^{0} G^{0}}\left(k^{2}\right)$ are replaced with the inverse free propagators of the Higgs boson $\Delta_{H}^{-1}(q)=q^{2}-M_{H}^{2}$ and the $G^{0}$ Goldstone boson $\Delta_{G^{0}}^{-1}(k)=k^{2}$, respectively, while $\hat{\Pi}_{\mu}^{Z G^{0}}(k)$ is substituted by $i M_{Z} k_{\mu}$, which represents the $G^{0} Z$ mixing. Of course, in the $R_{\xi}$ gauges there is no $G^{0} Z$ mixing at tree level because it cancels against the corresponding gauge-fixing term.

Within our Born-improved approximation the neutrinoexchange amplitude $\mathcal{T}_{t}\left(Z_{L} Z_{L}\right)$ retains its tree-level form; its only function is to provide the PT term $\mathcal{T}_{P}^{H}\left(Z_{L} Z_{L}\right)$. This latter term is responsible for the bad high-energy behavior of both the resonant and non-resonant amplitudes, which violate the ET separately. The validity of the ET for the individual amplitudes can be restored only after the PT term $\mathcal{T}_{P}^{H}\left(Z_{L} Z_{L}\right)$ is added to the $s$-channel amplitude $\overline{\mathcal{T}}_{s}^{H}\left(Z_{L} Z_{L}\right)$, exactly as happens in the case of the tree-level (nonresummed) $\hat{\mathcal{T}}_{s}^{H}\left(Z_{L} Z_{L}\right)$. Indeed, it is not difficult to show that the amplitudes $\overline{\mathcal{T}}_{s}^{H}\left(Z_{L} Z_{L}\right)+\mathcal{T}_{P}^{H}$ and $\mathcal{T}_{t}\left(Z_{L} Z_{L}\right)-\mathcal{T}_{P}^{H}$ satisfy the GET and hence the ET individually. For example, by employing the PT WI's in Eqs. (6.24)-(6.26), we have that

$$
\begin{aligned}
\overline{\mathcal{T}}_{s}^{H}\left(Z_{L} Z_{L}\right)+\mathcal{T}_{P}^{H}= & -\overline{\mathcal{T}}_{s}^{H}\left(G^{0} G^{0}\right)-i \overline{\mathcal{T}}_{s}^{H}\left(z G^{0}\right) \\
& -i \overline{\mathcal{T}}_{s}^{H}\left(G^{0} z\right)+\overline{\mathcal{T}}_{s}^{H}(z z),
\end{aligned}
$$

where 


$$
\begin{aligned}
\overline{\mathcal{T}}_{s}^{H}\left(G^{0} G^{0}\right)= & -i\left[\Gamma_{0}^{H G^{0} G^{0}}+\hat{\Gamma}^{H G^{0} G^{0}}\left(q, k_{1}, k_{2}\right)\right] \\
& \times \hat{\Delta}^{H}(q)\left(\frac{g_{w} m}{2 M_{W}}\right) \bar{v}\left(p_{2}\right) u\left(p_{1}\right),
\end{aligned}
$$

and the sum of the resummed amplitudes $\overline{\mathcal{T}}_{s}^{H}\left(z G^{0}\right)$ $+\overline{\mathcal{T}}_{s}^{H}\left(G^{0} z\right)$ is defined analogously to Eq. (6.19), i.e.,

$$
\begin{aligned}
\overline{\mathcal{T}}_{s}^{H}\left(z G^{0}\right)+\overline{\mathcal{T}}_{s}^{H}\left(G^{0} z\right) \\
=z^{\mu}\left(k_{1}\right) \overline{\mathcal{T}}_{s \mu}^{H}\left(Z G^{0}\right)+z^{\nu}\left(k_{2}\right) \overline{\mathcal{T}}_{s \nu}^{H}\left(G^{0} Z\right) \\
=-i\left\{z^{\mu}\left(k_{1}\right)\left[\Gamma_{0 \mu}^{H Z G^{0}}+\hat{\Gamma}_{\mu}^{H Z G^{0}}\left(q, k_{1}, k_{2}\right)\right]\right. \\
\left.+z^{\nu}\left(k_{2}\right)\left[\Gamma_{0 \nu}^{H G^{0} Z}+\hat{\Gamma}_{\nu}^{H G^{0} Z}\left(q, k_{1}, k_{2}\right)\right]\right\} \\
\quad \times\left(\frac{g_{w} m}{2 M_{W}}\right) \hat{\Delta}^{H}(q) \bar{v}\left(p_{2}\right) u\left(p_{1}\right) .
\end{aligned}
$$

Finally, we have defined $\overline{\mathcal{T}}_{s}^{H}(z z)$ $=z^{\mu}\left(k_{1}\right) z^{\nu}\left(k_{2}\right) \overline{\mathcal{T}}_{s \mu \nu}^{H}\left(Z_{L} Z_{L}\right)$. In the derivation of Eq. (6.27), we have also used the PT WI: $\hat{\Pi}_{\mu}^{Z G^{0}}(k)$ $=-i M_{Z} k_{\mu} \hat{\Pi}^{G^{0} G^{0}}\left(k^{2}\right) / k^{2}$.

The above considerations can be straightforwardly extended to processes involving the $H W W$ vertex, e.g., the reaction $t \bar{t} \rightarrow H^{*} \rightarrow W_{L}^{+} W_{L}^{-}$. As has been discussed in Sec. III, one has to extract from the $b$-quark exchange graph the PT term related to the Higgs-mediated part of the amplitude [cf. Eq. (3.11)]. Similarly, after adding the PT term $\mathcal{T}_{P}^{H}$ to the resummed Higgs-exchange amplitude $\overline{\mathcal{T}}_{s}^{H}\left(W_{L}^{+} W_{L}^{-}\right)$, we can show that

$$
\begin{aligned}
\overline{\mathcal{T}}_{s}^{H}\left(W_{L}^{+} W_{L}^{-}\right)+\mathcal{T}_{P}^{H}= & -\overline{\mathcal{T}}_{s}^{H}\left(G^{+} G^{-}\right)+\overline{\mathcal{T}}_{s}^{H}\left(w^{+} G^{-}\right) \\
& -\overline{\mathcal{T}}_{s}^{H}\left(G^{+} w^{-}\right)+\overline{\mathcal{T}}_{s}^{H}\left(w^{+} w^{-}\right),
\end{aligned}
$$

which is in agreement with GET in Eq. (6.5). Again, the derivation relies on effective one-loop PT WI's, which are the same as those naively deduced from the classical action in the Born approximation. In this case, the PT WI's pertaining to the $H(q) W^{+}\left(k_{+}\right) W^{-}\left(k_{-}\right)$vertex are given by

$$
\begin{aligned}
& k_{+}^{\mu} \hat{\Gamma}_{\mu \nu}^{H W^{+} W^{-}}\left(q, k_{+}, k_{-}\right)+M_{W} \hat{\Gamma}_{\nu}^{H G^{+} W^{-}}\left(q, k_{+}, k_{-}\right) \\
& =-\frac{i g_{w}}{2} \hat{\Pi}_{\nu}^{W^{-} G^{+}}\left(k_{-}\right), \\
& k_{-}^{\nu} \hat{\Gamma}_{\mu \nu}^{H W^{+} W^{-}}\left(q, k_{+}, k_{-}\right)-M_{W} \hat{\Gamma}_{\mu}^{H W^{+} G^{-}}\left(q, k_{+}, k_{-}\right) \\
& \quad=\frac{i g_{w}}{2} \hat{\Pi}_{\mu}^{W^{+} G^{-}}\left(k_{+}\right),
\end{aligned}
$$

$$
\begin{gathered}
k_{ \pm}^{\mu} \hat{\Gamma}_{\mu}^{H W^{ \pm} G^{\mp}}\left(q, k_{ \pm}, k_{\mp}\right) \pm M_{W} \hat{\Gamma}^{H G^{+} G^{-}}\left(q, k_{+}, k_{-}\right) \\
= \pm \frac{i g_{w}}{2}\left[\hat{\Pi}^{H H}\left(q^{2}\right)+\hat{\Omega}\left(k_{\mp}^{2}\right)\right], \\
k_{+}^{\mu} k_{-}^{\nu} \hat{\Gamma}_{\mu \nu}^{H W^{+} W^{-}}\left(q, k_{+}, k_{-}\right)+M_{W}^{2} \hat{\Gamma}^{H G^{+} G^{-}}\left(q, k_{+}, k_{-}\right) \\
=\frac{i g_{w} M_{W}}{2}\left[\hat{\Pi}^{H H}\left(q^{2}\right)+\hat{\Omega}\left(k_{+}^{2}\right)+\hat{\Omega}\left(k_{-}^{2}\right)\right] .
\end{gathered}
$$

The analytic form of the PT vertex $\hat{\Gamma}_{\mu \nu}^{H W^{+} W^{-}}\left(q, k_{+}, k_{-}\right)$is given in Appendix A. The one-loop PT vertices $\hat{\Gamma}_{\mu}^{H W^{ \pm} G^{\mp}}\left(q, k_{ \pm}, k_{\mp}\right)$ and $\hat{\Gamma}^{H G^{+} G^{-}}\left(q, k_{+}, k_{-}\right)$may be gained by using the WI's in Eqs. (6.31)-(6.34) and known expressions for the PT Higgs- and $G^{+}$-boson self-energies [26].

At this point we should note that the GET is still valid for the Higgs-mediated part of the amplitude even if we use the RGI expression for the resummed Higgs boson propagator $\hat{R}^{H}(s)$ defined in Sec. V C. Similarly, one can define the process-dependent RGI combinations involving, e.g., the $H W^{+} W^{-}$vertex:

$$
\begin{aligned}
& \hat{R}_{\mu \nu}^{H W^{+} W^{-}}\left(q, k_{+}, k_{-}\right)=\left(\frac{M_{W}^{2}}{g_{w}^{2}}\right) \frac{1}{g_{w} M_{W}} \bar{\Gamma}_{\mu \nu}^{H W^{+} W^{-}}\left(q, k_{+}, k_{-}\right), \\
& \hat{R}_{\mu}^{H W^{+} G^{-}}\left(q, k_{+}, k_{-}\right)=\left(\frac{M_{W}^{2}}{g_{w}^{2}}\right) \frac{1}{g_{w}} \bar{\Gamma}_{\mu}^{H W^{+} G^{-}}\left(q, k_{+}, k_{-}\right), \\
& \hat{R}^{H G^{+} G^{-}}\left(q, k_{+}, k_{-}\right)=\left(\frac{M_{W}^{2}}{g_{w}^{2}}\right) \frac{M_{W}}{g_{w}} \bar{\Gamma}^{H G^{+} G^{-}}\left(q, k_{+}, k_{-}\right) .
\end{aligned}
$$

As before, "barred" quantities denote the sum over the treelevel and one-loop PT vertices. The UV finite, RGI quantities $\hat{R}^{H}, \hat{R}^{G^{+}}, \hat{R}_{\mu \nu}^{H W^{+} W^{-}}, \hat{R}_{\mu}^{H W^{+} G^{-}}$and $\hat{R}^{H G^{+} G^{-}}$satisfy treelevel-type PT WI's in direct analogy to those given in Eqs. (6.31)-(6.34). In this formulation, any resummed transition amplitude can be written in terms of a product of RGI quantities, where the vertices are replaced by the respective $\hat{R}$ expressions. As a consequence of this formulation, the factors $K^{ \pm}$and $K^{0}$ retain their tree-level values after renormalization provided the wave-function renormalizations for the external Goldstone bosons are properly taken into account.

In summary, we have shown how the diagrammatic method based on the PT enables the decomposition of the amplitude into a resummed propagator-like amplitude and a non-resonant background which satisfy the GET as well as the ET individually. This feature provides an additional nontrivial consistency check for the PT resummation approach, and, at the same time, renders the ET conceptually more intuitive.

\section{DISCUSSION AND CONCLUSIONS}

In this paper the formulation of the PT resummation approach has been extended to analyze resonant transition amplitudes which involve the SM Higgs boson as an interme- 


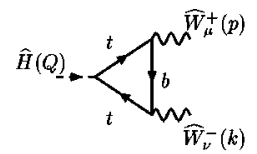

(a)

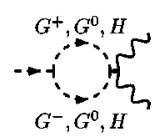

(b3)

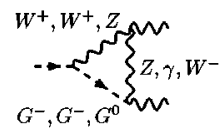

(c2)

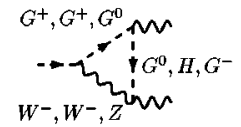

(c5)

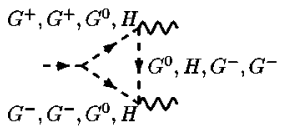

(c8)

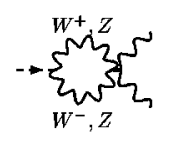

(b1)

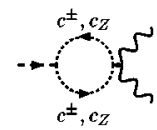

(b4)

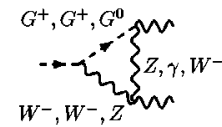

(c3)

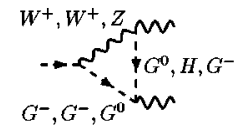

(c6)

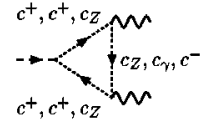

(c9)

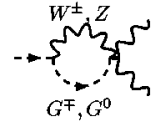

(b2)

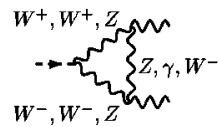

(c1)

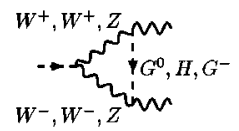

(c4)

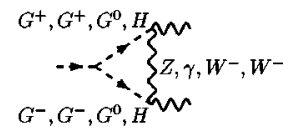

(c7)

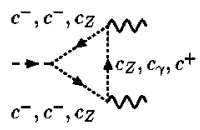

(c10)
FIG. 7. Graphs contributing to the absorptive part of the $H W W$ coupling in the BFG.

diate state. The main results of our study may be summarized as follows:

The PT rearrangement of the amplitude gives rise to a self-energy for the Higgs boson which is independent of the GFP in every gauge-fixing scheme. This selfenergy is universal, in the sense that it is process independent, and may be resummed following the method presented in Ref. [18]. In addition, it only displays physical fermionic and bosonic thresholds, in contrast to the gauge-dependent self-energies obtained by the conventional methods, where unphysical bosonic thresholds appear. Furthermore, it satisfies individually the OT, both for fermionic as well as bosonic contributions.

(ii) When the resummed Higgs boson propagator is multiplied by the universal quantity $g_{w}^{2} / M_{W}^{2}$, or, equivalently, by the inverse square of the vacuum expectation value of the Higgs field, it gives rise to a renormalization-group-invariant quantity, in direct analogy to the effective charge of the photon in QED. The above construction becomes possible by virtue of the naive, tree-level WI's satisfied by the GFPindependent PT sub-amplitudes.

(iii) At high energies any amplitude involving longitudinally polarized gauge bosons satisfies the ET, but the individual $s$-channel and $t$-channel contributions of the amplitude do not. Instead, the PT decomposition of such an amplitude gives rise to two kinematically distinct pieces, a genuine $s$-channel and a genuine $t$ channel, which satisfy the ET individually. Most im-

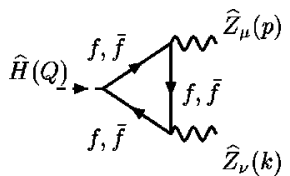

(a)

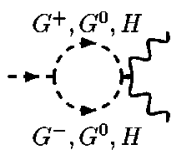

(b3)

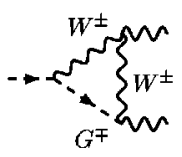

(c2)

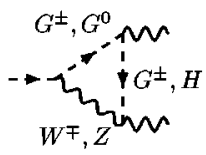

(c5)

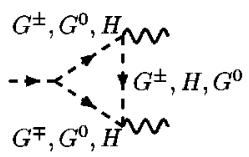

(c8)

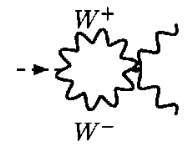

(b1)

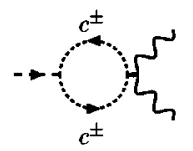

(b4)

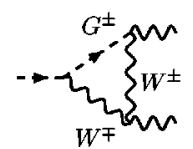

(c3)

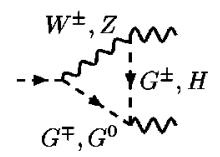

(c6)

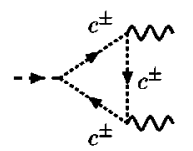

(c9)

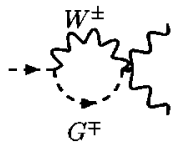

(b2)

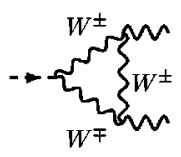

(c1)

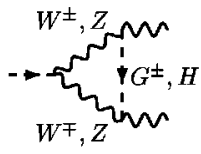

(c4)

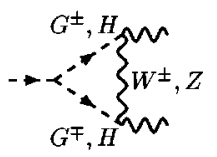

(c7)

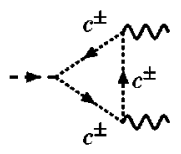

(c10)
FIG. 8. Diagrams contributing to the absorptive part of the $H Z Z$ coupling in the BFG.

portantly, the above property persists even after the $s$-channel Higgs boson self-energy has been resummed, thus solving a long-standing problem.

At this stage, it may also be worth reviewing briefly the state of affairs of the PT resummation approach. In particular, we wish to draw the attention of the reader to a number of significant developments as well as open issues related to this field, which have been extensively discussed in the recent literature and are also relevant to our study of the Higgsboson resonance. An important issue which remained unanswered for some time was whether the PT self-energy obtained by extracting pieces from the vertices and boxes can be resummed in a Dyson series, and whether the resummed PT propagator exhibits the same gauge-invariant pole as that calculated in the conventional perturbative framework. Both questions have been addressed in detail in [18]; it has been found that the PT self-energies are indeed resummable, and that the position of the pole remains unchanged. The proof presented in [18] was more involved than the standard arguments leading to the resummability of a conventional self-energy, and relied on a detailed diagrammatic analysis to all orders in perturbation theory. The resummability of the PT self-energies is a crucial ingredient for the construction of the corresponding effective charges. Independent studies have tested the latter property of the PT self-energies up to two loops for the $W^{ \pm}$and $Z$ bosons in the SM [20]. 
Up to date the PT has been applied only at the level of one-loop perturbation theory, and its extension to higher orders is still an important open issue. It would be interesting for example to establish whether the correspondence between PT and BFM at $\xi_{Q}=1$ persists beyond one loop. Furthermore, generalized versions of the PT proposed recently [30] may shed light on the structure of new background-field quantum actions even beyond one loop [43]. In addition, the diagrammatic understanding achieved by means of the PT and its possible extensions provides new insights into a variety of issues related to gauge independence, unitarity, and the ET, and exposes properties which are not manifest in the correlation functions derived with path-integral variational methods.

The significance of the analysis presented in this paper when computing the theoretical predictions for the Higgsboson lineshape is clear. The Born-improved amplitudes constructed with the above formalism are in accordance with all physical requirements imposed, and reliably capture the underlying dynamics. Most noticeably, the ability to construct a universal Higgs-mediated component, in direct analogy to the QED effective charge, is rather intriguing. This universal part is common to every Higgs-boson-mediated process, and, even though the process-dependent background must be eventually taken into account, it determines the Higgs-boson lineshape comfortably away from the resonance. It would be of great phenomenological importance to confront the predictions for Higgs-production and decay processes computed within the PT resummation approach against future data obtained from planned high-energy colliders such as the LHC, the next-linear $e^{+} e^{-}$collider with c.m. energy $500 \mathrm{GeV}$, and the first muon collider.

\section{ACKNOWLEDGMENTS}

The work of J.P. is funded by a Marie Curie Fellowship (TMR-ERBFMBICT 972024). J.P. also acknowledges financial support from the Department of Physics and Astronomy of the University of Manchester (PPARC grant), and the Center de Physique Théorique (CPT) at Marseille (CNRS grant), while parts of this work were being completed, and thanks the Max Planck Institute for its warm hospitality. A.P. thanks the theory group of Manchester University for the kind hospitality extended to him.

\section{APPENDIX A: ONE-LOOP ABSORPTIVE $H W W$ COUPLING IN THE PT}

Based on the established equivalence between the PT and the covariant background field gauge for $\xi_{Q}=1$, we calculate the absorptive part of the effective PT $H W W$ vertex at one loop, using the Feynman rules listed in [28]. The analytic results are expressed in terms of standard loop integrals introduced by 't Hooft and Veltman [23]. For definiteness, we use the conventions of Ref. [24].

If one assumes that the external $W$ bosons are contracted with physical polarization vectors or conserved currents, the one-loop PT $H W W$ coupling $\hat{\Gamma}_{\mu \nu}^{H W^{+} W^{-}}\left(q, k_{+}, k_{-}\right)$may then be decomposed in general as follows:

$$
\hat{\Gamma}_{\mu \nu}^{H W^{+} W^{-}}(Q, p, k)=g_{w} M_{W}\left[\left(1+A\left(Q^{2}\right)\right) g_{\mu \nu}+B\left(Q^{2}\right) \frac{k_{\mu} p_{\nu}}{M_{W}^{2}}+i C\left(Q^{2}\right) \frac{1}{M_{W}^{2}} \varepsilon_{\mu \nu \lambda \rho} k^{\lambda} p^{\rho}\right],
$$

where $Q+p+k=0$ and $A\left(Q^{2}\right), B\left(Q^{2}\right)$ and $C\left(Q^{2}\right)$ are general form-factors. Only $A\left(Q^{2}\right)$ must be renormalized, whereas $B\left(Q^{2}\right)$ and $C\left(Q^{2}\right)$ are UV finite. The form-factor $C\left(Q^{2}\right)$ occurs in $C P$-violating scenarios only, i.e., $C\left(Q^{2}\right)=0$.

In the improved Born-level approximation, only the absorptive parts of the form-factors $A\left(Q^{2}\right)$ and $B\left(Q^{2}\right)$ are of relevance, as the dispersive parts participate in the one-loop renormalization. The diagrams contributing to the absorptive form-factors $\bar{A}$ and $\bar{B}$ are shown in Fig. 7. To a good approximation, the external $W$ bosons are considered to be stable and the $b$ quark massless. The analytic results for the absorptive form-factor $\bar{A}\left(Q^{2}\right)$ are then given by

$$
\begin{aligned}
& i \bar{A}_{(a)}=\frac{\alpha_{w}}{16 \pi} \frac{m_{t}^{2}}{M_{W}^{2}}\left[8 \bar{C}_{24}+\left(Q^{2}+k^{2}-p^{2}+4 m_{t}^{2}\right) \bar{C}_{0}+\left(3 Q^{2}-3 k^{2}-p^{2}\right) \bar{C}_{11}+\left(Q^{2}+5 k^{2}-p^{2}\right) \bar{C}_{12}\right]\left(m_{t}^{2}, 0, m_{t}^{2}\right), \\
& i \bar{A}_{(b 1)}=-\frac{\alpha_{w}}{\pi}\left[\bar{B}_{0}\left(Q^{2}, M_{W}^{2}, M_{W}^{2}\right)+2 \frac{M_{W}^{2}}{M_{Z}^{2}} \bar{B}_{0}\left(Q^{2}, M_{Z}^{2}, M_{Z}^{2}\right)\right], \\
& i \bar{A}_{(b 2)}=i \bar{A}_{(c 5)}=i \bar{A}_{(c 6)}=0, \\
& i \bar{A}_{(b 3)}=-\frac{\alpha_{w}}{16 \pi}\left[\left(\frac{M_{H}^{2}}{M_{W}^{2}}+2\right) \bar{B}_{0}\left(Q^{2}, M_{W}^{2}, M_{W}^{2}\right)+\frac{1}{2}\left(\frac{M_{H}^{2}}{M_{W}^{2}}+2 \frac{M_{Z}^{2}}{M_{W}^{2}}\right) \bar{B}_{0}\left(Q^{2}, M_{Z}^{2}, M_{Z}^{2}\right)+\frac{3}{2} \frac{M_{H}^{2}}{M_{W}^{2}} \bar{B}_{0}\left(Q^{2}, M_{H}^{2}, M_{H}^{2}\right)\right], \\
& i \bar{A}_{(b 4)}=\frac{\alpha_{w}}{2 \pi}\left[\bar{B}_{0}\left(Q^{2}, M_{W}^{2}, M_{W}^{2}\right)+\bar{B}_{0}\left(Q^{2}, M_{Z}^{2}, M_{Z}^{2}\right)\right],
\end{aligned}
$$




$$
\begin{aligned}
i \bar{A}_{(c 1)}= & \frac{\alpha_{w}}{\pi}\left[\left(1-\frac{M_{W}^{2}}{M_{Z}^{2}}\right)\left(4 \bar{C}_{24}+\left(Q^{2}-k^{2}-p^{2}\right) \bar{C}_{0}\right)\left(M_{W}^{2}, 0, M_{W}^{2}\right)+\frac{M_{W}^{2}}{M_{Z}^{2}}\left(4 \bar{C}_{24}+\left(Q^{2}-k^{2}-p^{2}\right) \bar{C}_{0}\right)\left(M_{W}^{2}, M_{Z}^{2}, M_{W}^{2}\right)\right. \\
& \left.+\frac{M_{Z}^{2}}{M_{W}^{2}}\left(4 \bar{C}_{24}+\left(Q^{2}-k^{2}-p^{2}\right) \bar{C}_{0}\right)\left(M_{Z}^{2}, M_{W}^{2}, M_{Z}^{2}\right)\right], \\
i \bar{A}_{(c 2)}+i \bar{A}_{(c 3)}= & \frac{\alpha_{w}}{2 \pi} Q^{2}\left[\left(2 \frac{M_{W}^{2}}{M_{Z}^{2}}-1\right) \bar{C}_{0}\left(M_{W}^{2}, M_{Z}^{2}, M_{W}^{2}\right)+\left(2 \frac{M_{W}^{2}}{M_{Z}^{2}}-1\right) \bar{C}_{0}\left(M_{W}^{2}, 0, M_{W}^{2}\right)+\bar{C}_{0}\left(M_{Z}^{2}, M_{W}^{2}, M_{Z}^{2}\right)\right], \\
i \bar{A}_{(c 4)}= & -\frac{\alpha_{w}}{4 \pi}\left[\frac{M_{Z}^{4}}{M_{W}^{2}}\left(2 \frac{M_{W}^{2}}{M_{Z}^{2}}-1\right)^{2} \bar{C}_{0}\left(M_{Z}^{2}, M_{W}^{2}, M_{Z}^{2}\right)+M_{W}^{2} \bar{C}_{0}\left(M_{W}^{2}, M_{Z}^{2}, M_{W}^{2}\right)+M_{W}^{2} \bar{C}_{0}\left(M_{W}^{2}, M_{H}^{2}, M_{W}^{2}\right)\right], \\
i \bar{A}_{(c 7)=} & -\frac{\alpha_{w}}{8 \pi}\left[M_{Z}^{2}\left(\frac{M_{H}^{2}}{M_{W}^{2}}+2\right)\left(2 \frac{M_{W}^{2}}{M_{Z}^{2}}-1\right)^{2} \bar{C}_{0}\left(M_{W}^{2}, M_{Z}^{2}, M_{W}^{2}\right)+4 M_{W}^{2}\left(\frac{M_{H}^{2}}{M_{W}^{2}}+2\right)\left(1-\frac{M_{W}^{2}}{M_{Z}^{2}}\right) \bar{C}_{0}\left(M_{W}^{2}, 0, M_{W}^{2}\right)\right. \\
& \left.+M_{W}^{2}\left(\frac{M_{H}^{2}}{M_{W}^{2}}+2 \frac{M_{Z}^{2}}{M_{W}^{2}}\right) \bar{C}_{0}\left(M_{Z}^{2}, M_{W}^{2}, M_{Z}^{2}\right)+3 M_{H}^{2} \bar{C}_{0}\left(M_{H}^{2}, M_{W}^{2}, M_{H}^{2}\right)\right], \\
i \bar{A}_{(c 8)}= & -\frac{\alpha_{w}}{8 \pi}\left[\left(\frac{M_{H}^{2}}{M_{W}^{2}}+2\right)\left(\bar{C}_{24}\left(M_{W}^{2}, M_{Z}^{2}, M_{W}^{2}\right)+\bar{C}_{24}\left(M_{W}^{2}, M_{H}^{2}, M_{W}^{2}\right)\right)+\left(\frac{M_{H}^{2}}{M_{W}^{2}}+2 \frac{M_{Z}^{2}}{M_{W}^{2}}\right) \bar{C}_{24}\left(M_{Z}^{2}, M_{W}^{2}, M_{Z}^{2}\right)\right. \\
& \left.+3 \frac{M_{H}^{2}}{M_{W}^{2}} \bar{C}_{24}\left(M_{H}^{2}, M_{W}^{2}, M_{H}^{2}\right)\right], \\
i \bar{A}_{(c 9)+}+i \bar{A}_{(c 10)=}= & \frac{2 \alpha_{w}}{\pi}\left[\frac{M_{W}^{2}}{M_{Z}^{2}} \bar{C}_{24}\left(M_{W}^{2}, M_{Z}^{2}, M_{W}^{2}\right)+\left(1-\frac{M_{W}^{2}}{M_{Z}^{2}}\right) \bar{C}_{24}\left(M_{W}^{2}, 0, M_{W}^{2}\right)+\bar{C}_{24}\left(M_{Z}^{2}, M_{W}^{2}, M_{Z}^{2}\right)\right] .
\end{aligned}
$$

Here and in the following, we do not display the first three arguments of the $C$ functions $\left(p^{2}, k^{2}, Q^{2}\right)$, which are common. The bar on the loop functions symbolizes that only the absorptive part should be considered.

Similarly, the individual contributions to the absorptive form-factor $\bar{B}\left(Q^{2}\right)$ are found to give

$$
\begin{aligned}
i \bar{B}_{(a)}= & -\frac{\alpha_{w}}{8 \pi} m_{t}^{2}\left(4 \bar{C}_{23}+\bar{C}_{0}+3 \bar{C}_{11}+\bar{C}_{12}\right)\left(m_{t}^{2}, 0, m_{t}^{2}\right) \\
i \bar{B}_{(b 1)}= & i \bar{B}_{(b 2)}=i \bar{B}_{(b 3)}=i \bar{B}_{(b 4)}=i \bar{B}_{(c 4)}=i \bar{B}_{(c 7)}=0 \\
i \bar{B}_{(c 1)}= & \frac{2 \alpha_{w}}{\pi}\left[M_{W}^{2}\left(1-\frac{M_{W}^{2}}{M_{Z}^{2}}\right)\left(2 \bar{C}_{11}+2 \bar{C}_{23}+\bar{C}_{0}\right)\left(M_{W}^{2}, 0, M_{W}^{2}\right)+\frac{M_{W}^{4}}{M_{Z}^{2}}\left(2 \bar{C}_{11}+2 \bar{C}_{23}+\bar{C}_{0}\right)\left(M_{W}^{2}, M_{Z}^{2}, M_{W}^{2}\right)\right. \\
& \left.+M_{W}^{2}\left(2 \bar{C}_{11}+2 \bar{C}_{23}+\bar{C}_{0}\right)\left(M_{Z}^{2}, M_{W}^{2}, M_{Z}^{2}\right)\right] \\
i \bar{B}_{(c 2)}+i \bar{B}_{(c 3)}= & \frac{\alpha_{w}}{2 \pi}\left[M_{W}^{2}\left(2 \frac{M_{W}^{2}}{M_{Z}^{2}}-1\right)\left(\bar{C}_{0}+\bar{C}_{11}+\bar{C}_{12}\right)\left(M_{W}^{2}, M_{Z}^{2}, M_{W}^{2}\right)+2 M_{W}^{2}\left(1-\frac{M_{W}^{2}}{M_{Z}^{2}}\right)\right. \\
& \left.\times\left(\bar{C}_{0}+\bar{C}_{11}+\bar{C}_{12}\right)\left(M_{W}^{2}, 0, M_{W}^{2}\right)+M_{W}^{2}\left(\bar{C}_{0}+\bar{C}_{11}+\bar{C}_{12}\right)\left(M_{Z}^{2}, M_{W}^{2}, M_{Z}^{2}\right)\right] \\
& i \bar{B}_{(c 5)}+i \bar{B}_{(c 6)}= \\
& \frac{\alpha_{w}}{4 \pi}\left[\left(M_{Z}^{2}-2 M_{W}^{2}\right)\left(\bar{C}_{0}+\bar{C}_{12}-\bar{C}_{11}\right)\left(M_{Z}^{2}, M_{W}^{2}, M_{Z}^{2}\right)-M_{W}^{2}\left(\bar{C}_{0}+\bar{C}_{12}-\bar{C}_{11}\right)\left(M_{W}^{2}, M_{Z}^{2}, M_{W}^{2}\right)\right. \\
& \left.+M_{W}^{2}\left(\bar{C}_{0}+\bar{C}_{12}-\bar{C}_{11}\right)\left(M_{W}^{2}, M_{H}^{2}, M_{W}^{2}\right)\right],
\end{aligned}
$$




$$
\begin{aligned}
i \bar{B}_{(c 8)}= & \frac{\alpha_{w}}{8 \pi}\left\{\left(M_{H}^{2}+2 M_{W}^{2}\right)\left[\left(\bar{C}_{11}+\bar{C}_{23}\right)\left(M_{W}^{2}, M_{Z}^{2}, M_{W}^{2}\right)+\left(\bar{C}_{11}+\bar{C}_{23}\right)\left(M_{W}^{2}, M_{H}^{2}, M_{W}^{2}\right)\right]\right. \\
& \left.+\left(M_{H}^{2}+2 M_{Z}^{2}\right)\left(\bar{C}_{11}+\bar{C}_{23}\right)\left(M_{Z}^{2}, M_{W}^{2}, M_{Z}^{2}\right)+3 M_{H}^{2}\left(\bar{C}_{11}+\bar{C}_{23}\right)\left(M_{H}^{2}, M_{W}^{2}, M_{H}^{2}\right)\right\}, \\
i \bar{B}_{(c 9)}+i \bar{B}_{(c 10)}= & -\frac{2 \alpha_{w}}{\pi}\left[\frac{M_{W}^{4}}{M_{Z}^{2}}\left(\bar{C}_{11}+\bar{C}_{23}\right)\left(M_{W}^{2}, M_{Z}^{2}, M_{W}^{2}\right)+M_{W}^{2}\left(1-\frac{M_{W}^{2}}{M_{Z}^{2}}\right)\left(\bar{C}_{11}+\bar{C}_{23}\right)\left(M_{W}^{2}, 0, M_{W}^{2}\right)\right. \\
& \left.+M_{W}^{2}\left(\bar{C}_{11}+\bar{C}_{23}\right)\left(M_{Z}^{2}, M_{W}^{2}, M_{Z}^{2}\right)\right] .
\end{aligned}
$$

\section{APPENDIX B: ONE-LOOP ABSORPTIVE HZZ COUPLING IN THE PT}

Here we present the one-loop results for the absorptive form-factors $\bar{A}$ and $\bar{B}$ of the $H Z Z$ coupling in terms of standard loop integrals. We consider the general decomposition of the one-loop $H Z Z$ vertex

$$
\hat{\Gamma}_{\mu \nu}^{H Z Z}(Q, p, k)=\frac{g_{w}}{c_{w}} M_{Z}\left[\left(1+A\left(Q^{2}\right)\right) g_{\mu \nu}+B\left(Q^{2}\right) \frac{k_{\mu} p_{\nu}}{M_{Z}^{2}}\right],
$$

where the CP-violating form-factor $C\left(Q^{2}\right)$ analogous to Eq. (A1) is absent at one-loop in the SM. In particular, we are interested in the absorptive part of the form-factors $A\left(Q^{2}\right)$ and $B\left(Q^{2}\right)$. Calculating the graphs shown in Fig. 8, we obtain

$$
\begin{aligned}
i \bar{A}_{(a)}= & \frac{\alpha_{w}}{16 \pi} \frac{m_{t}^{2}}{M_{W}^{2}}\left\{\left(g_{L}^{2}+g_{R}^{2}\right)\left[8 \bar{C}_{24}+\left(Q^{2}+k^{2}-p^{2}+4 m_{t}^{2}\right) \bar{C}_{0}+\left(3 Q^{2}-3 k^{2}-p^{2}\right) \bar{C}_{11}+\left(Q^{2}+5 k^{2}-p^{2}\right) \bar{C}_{12}\right]\right. \\
& \left.\times\left(m_{t}^{2}, m_{t}^{2}, m_{t}^{2}\right)+2 g_{L} g_{R}\left[4 m_{t}^{2} \bar{C}_{0}+\left(Q^{2}-k^{2}+p^{2}\right) \bar{C}_{11}+\left(Q^{2}+k^{2}-p^{2}\right) \bar{C}_{12}\right]\left(m_{t}^{2}, m_{t}^{2}, m_{t}^{2}\right)\right\}, \\
i \bar{A}_{(b 1)}= & -\frac{2 \alpha_{w}}{\pi} \frac{M_{W}^{4}}{M_{Z}^{4}} \bar{B}_{0}\left(Q^{2}, M_{W}^{2}, M_{W}^{2}\right), \\
i \bar{A}_{(b 2)}= & i \bar{A}_{(c 5)}=i \bar{A}_{(c 6)}=0, \\
i \bar{A}_{(b 3)}= & -\frac{\alpha_{w}}{16 \pi}\left[\left(\frac{M_{H}^{2}}{M_{W}^{2}}+2\right)\left(2 \frac{M_{W}^{2}}{M_{Z}^{2}}-1\right)^{2} \bar{B}_{0}\left(Q^{2}, M_{W}^{2}, M_{W}^{2}\right)+\frac{1}{2}\left(\frac{M_{H}^{2}}{M_{W}^{2}}+2 \frac{M_{Z}^{2}}{M_{W}^{2}}\right) \bar{B}_{0}\left(Q^{2}, M_{Z}^{2}, M_{Z}^{2}\right)\right. \\
& \left.+\frac{3}{2} \frac{M_{H}^{2}}{M_{W}^{2}} \bar{B}_{0}\left(Q^{2}, M_{H}^{2}, M_{H}^{2}\right)\right], \\
i \bar{A}_{(b 4)}= & \frac{\alpha_{w}}{\pi} \frac{M_{W}^{4}}{M_{Z}^{4}} \bar{B}_{0}\left(Q^{2}, M_{W}^{2}, M_{W}^{2}\right), \\
i \bar{A}_{(c 1)}= & \frac{2 \alpha_{w}}{\pi} \frac{M_{W}^{4}}{M_{Z}^{4}}\left[4 \bar{C}_{24}+\left(Q^{2}-k^{2}-p^{2}\right) \bar{C}_{0}\right]\left(M_{W}^{2}, M_{W}^{2}, M_{W}^{2}\right), \\
i \bar{A}_{(c 7)}= & -\frac{\alpha_{w}}{8 \pi}\left[2\left(M_{H}^{2}+2 M_{W}^{2}\right) \bar{C}_{0}\left(M_{W}^{2}, M_{W}^{2}, M_{W}^{2}\right)+3 M_{Z}^{2} \frac{M_{H}^{2}}{M_{W}^{2}} \bar{C}_{0}\left(M_{H}^{2}, M_{Z}^{2}, M_{H}^{2}\right)\right], \\
i \bar{A}_{(c 4)}= & -\frac{\alpha_{w}}{4 \pi}\left[2 M_{W}^{2} \bar{C}_{0}\left(M_{W}^{2}, M_{W}^{2}, M_{W}^{2}\right)+\frac{M_{Z}^{4}}{M_{W}^{2}} \bar{C}_{0}\left(M_{Z}^{2}, M_{H}^{2}, M_{Z}^{2}\right)\right], \\
i \bar{A}_{(c 3)}= & -\frac{\alpha_{w}}{2 \pi} \frac{M_{W}^{2}}{M_{Z}^{2}} Q^{2} \bar{C}_{0}\left(M_{W}^{2}, M_{W}^{2}, M_{W}^{2}\right), \\
&
\end{aligned}
$$




$$
\begin{aligned}
i \bar{A}_{(c 8)}= & -\frac{\alpha_{w}}{4 \pi}\left[\left(2 \frac{M_{W}^{2}}{M_{Z}^{2}}-1\right)^{2}\left(\frac{M_{H}^{2}}{M_{W}^{2}}+2\right) \bar{C}_{24}\left(M_{W}^{2}, M_{W}^{2}, M_{W}^{2}\right)+\frac{1}{2}\left(\frac{M_{H}^{2}}{M_{W}^{2}}+2 \frac{M_{Z}^{2}}{M_{W}^{2}}\right) \bar{C}_{24}\left(M_{Z}^{2}, M_{H}^{2}, M_{Z}^{2}\right)\right. \\
& \left.+\frac{3}{2} \frac{M_{H}^{2}}{M_{W}^{2}} \bar{C}_{24}\left(M_{H}^{2}, M_{Z}^{2}, M_{H}^{2}\right)\right], \\
i \bar{A}_{(c 9)}+i \bar{A}_{(c 10)}= & \frac{4 \alpha_{w}}{\pi} \frac{M_{W}^{4}}{M_{Z}^{4}} \bar{C}_{24}\left(M_{W}^{2}, M_{W}^{2}, M_{W}^{2}\right) .
\end{aligned}
$$

In Eq. (B2), we have defined as $g_{L}=\left(2 M_{W}^{2} / M_{Z}^{2}\right)-1$ and $g_{R}=-2\left(1-M_{W}^{2} / M_{Z}^{2}\right)$.

Furthermore, the individual contributions to the $B$ form-factor are given by

$$
\begin{aligned}
& i \bar{B}_{(a)}=-\frac{\alpha_{w}}{8 \pi} M_{Z}^{2} \frac{m_{t}^{2}}{M_{W}^{2}}\left\{\left(g_{L}^{2}+g_{R}^{2}\right)\left(4 \bar{C}_{23}+\bar{C}_{0}+3 \bar{C}_{11}+\bar{C}_{12}\right)\left(m_{t}^{2}, m_{t}^{2}, m_{t}^{2}\right)+2 g_{L} g_{R}\left(\bar{C}_{11}-\bar{C}_{12}\right)\left(m_{t}^{2}, m_{t}^{2}, m_{t}^{2}\right)\right\}, \\
& i \bar{B}_{(b 1)}=i \bar{B}_{(b 2)}=i \bar{B}_{(b 3)}=i \bar{B}_{(b 4)}=i \bar{B}_{(c 4)}=i \bar{B}_{(c 7)}=0 \\
& i \bar{B}_{(c 1)}=\frac{4 \alpha_{w}}{\pi} \frac{M_{W}^{4}}{M_{Z}^{2}}\left(2 \bar{C}_{11}+2 \bar{C}_{23}+\bar{C}_{0}\right)\left(M_{W}^{2}, M_{W}^{2}, M_{W}^{2}\right), \\
& i \bar{B}_{(c 2)}+i \bar{B}_{(c 3)}=-\frac{\alpha_{w}}{2 \pi} M_{W}^{2}\left(\bar{C}_{0}+\bar{C}_{11}+\bar{C}_{12}\right)\left(M_{W}^{2}, M_{W}^{2}, M_{W}^{2}\right), \\
& i \bar{B}_{(c 5)}+i \bar{B}_{(c 6)}=\frac{\alpha_{w}}{4 \pi}\left[2\left(2 M_{W}^{2}-M_{Z}^{2}\right)\left(\bar{C}_{0}+\bar{C}_{12}-\bar{C}_{11}\right)\left(M_{W}^{2}, M_{W}^{2}, M_{W}^{2}\right)-\frac{M_{Z}^{4}}{M_{W}^{2}}\left(\bar{C}_{0}+\bar{C}_{12}-\bar{C}_{11}\right)\left(M_{Z}^{2}, M_{H}^{2}, M_{Z}^{2}\right)\right], \\
& i \bar{B}_{(c 8)}=\frac{\alpha_{w}}{4 \pi} M_{Z}^{2}\left[\left(2 \frac{M_{W}^{2}}{M_{Z}^{2}}-1\right)^{2}\left(\frac{M_{H}^{2}}{M_{W}^{2}}+2\right)\left(\bar{C}_{11}+\bar{C}_{23}\right)\left(M_{W}^{2}, M_{W}^{2}, M_{W}^{2}\right)+\frac{1}{2}\left(\frac{M_{H}^{2}}{M_{W}^{2}}+2 \frac{M_{Z}^{2}}{M_{W}^{2}}\right)\left(\bar{C}_{11}+\bar{C}_{23}\right)\right. \\
& \left.\times\left(M_{Z}^{2}, M_{H}^{2}, M_{Z}^{2}\right)+\frac{3}{2} \frac{M_{H}^{2}}{M_{W}^{2}}\left(\bar{C}_{11}+\bar{C}_{23}\right)\left(M_{H}^{2}, M_{Z}^{2}, M_{H}^{2}\right)\right], \\
& i \bar{B}_{(c 9)}+i \bar{B}_{(c 10)}=-\frac{4 \alpha_{w}}{\pi} \frac{M_{W}^{4}}{M_{Z}^{2}}\left(\bar{C}_{11}+\bar{C}_{23}\right)\left(M_{W}^{2}, M_{W}^{2}, M_{W}^{2}\right) .
\end{aligned}
$$

[1] P. W. Higgs, Phys. Rev. Lett. 12, 132 (1964); P. W. Higgs, Phys. Rev. 145, 1156 (1966); F. Englert and R. Brout, Phys. Rev. Lett. 13, 321 (1964); G. S. Guralnik, C. R. Hagen, and T. W. Kibble, ibid. 13, 585 (1964).

[2] J. F. Grivaz, Proceedings of the International EPS Conference on High-Energy Physics, Brussels, 1995.

[3] N. Cabibbo, L. Maiani, G. Parisi, and R. Petronzio, Nucl. Phys. B158, 295 (1979); M. Chanowitz, M. Furman, and I. Hinchliffe, Phys. Lett. 78B, 285 (1978); R. A. Flores and M. Sher, Phys. Rev. D 27, 1679 (1983); M. Lindner, Z. Phys. C 31, 295 (1986); M. Sher, Phys. Rep. 179, 273 (1989); M. Lindner, M. Sher, and H. W. Zaglauer, Phys. Lett. B 228, 139 (1989).

[4] A. Hasenfratz, K. Jansen, C. Lang, T. Neuhaus, and H. Yoneyama, Phys. Lett. B 199, 531 (1987); J. Kuti, L. Liu, and Y.
Shen, Phys. Rev. Lett. 61, 678 (1988); M. Lüscher and P. Weisz, Nucl. Phys. B318, 705 (1989).

[5] M. Veltman, Nucl. Phys. B123, 89 (1977).

[6] A. Blondel, talk at the ICHEP 96, Warsaw, 1996, and the LEP Electroweak Working Group, Internal Note LEPEWWG/9602 .

[7] G. Degrassi, P. Gambino, M. Passera, and A. Sirlin, Phys. Lett. B 418, 209 (1998).

[8] An even more stringent upper bound of about $250 \mathrm{GeV}$ has been presented in two recent talks: J. Mnich, "LEP Physics-An Overview,' talk given at The Sixth International Symposium on Particles, Strings and Cosmology, Boston, MA, 1988; J. J. Blaising, "Higgs Mass from Electroweak Data," talk given at the XXXIIIth Rencontres de Moriond, Les Arcs, France, 1998. 
[9] M. Carena and P. M. Zerwas, Conveners, "Higgs Physics at LEP-II," CERN Yellow Report, CERN-96-01, hep-ph/9602250.

[10] J. D. Bjorken, Proceedings of the Summer Institute on Particle Physics, SLAC Report 198 (1976); J. Ellis, M. K. Gaillard, and D. V. Nanopoulos, Nucl. Phys. B106, 292 (1976); B. W. Lee, C. Quigg, and H. B. Thacker, Phys. Rev. D 16, 1519 (1977); B. L. Ioffe and V. A. Khoze, Sov. J. Part. Nucl. 9, 50 (1978).

[11] A. Sirlin, Phys. Rev. Lett. 67, 2127 (1991); Phys. Lett. B 267, 240 (1991); M. Nowakowski and A. Pilaftsis, Z. Phys. C 60, 121 (1993); U. Baur and D. Zeppenfeld, Phys. Rev. Lett. 75, 1002 (1995); M. Beuthe, R. Gonzalez Felipe, G. Lopez Castro, and J. Pestieau, Nucl. Phys. B498, 55 (1997).

[12] D. A. Dicus and V. S. Mathur, Phys. Rev. D 7, 3111 (1973); W. W. Repko and C. J. Suchyta III, Phys. Rev. Lett. 62, 859 (1989); S. Dawson and S. Willenbrock, Phys. Rev. D 40, 2880 (1989); A. Dobado, Phys. Lett. B 237, 457 (1990); G. Valencia and S. Willenbrock, Phys. Rev. D 46, 2247 (1992); M. H. Seymour, Phys. Lett. B 354, 409 (1995); A. Ghinculov and J. J. van der Bij, Nucl. Phys. B482, 59 (1996).

[13] J. M. Cornwall, D. N. Levin, and G. Tiktopoulos, Phys. Rev. D 10, 1145 (1974); Phys. Rev. E 11, 972 (1975); C. E. Vayonakis, Lett. Nuovo Cimento 17, 383 (1976).

[14] M. S. Chanowitz and M. K. Gaillard, Nucl. Phys. B261, 379 (1985); G. J. Gounaris, R. Kögerler, and H. Neufeld, Phys. Rev. D 34, 3257 (1986).

[15] J. M. Cornwall, in Proceedings of the French-American Seminar on Theoretical Aspects of Quantum Chromodynamics, Marseille, France, 1981, edited J. W. Dash (Centre de Physique Théorique, Marseille, 1982).

[16] J. M. Cornwall, Phys. Rev. D 26, 1453 (1982).

[17] J. M. Cornwall and J. Papavassiliou, Phys. Rev. D 40, 3474 (1989); G. Degrassi and A. Sirlin, ibid. 46, 3104 (1992).

[18] J. Papavassiliou and A. Pilaftsis, Phys. Rev. Lett. 75, 3060 (1995); Phys. Rev. D 53, 2128 (1996).

[19] J. Papavassiliou and A. Pilaftsis, Phys. Rev. D 54, 5315 (1996).

[20] K. Philippides and A. Sirlin, Phys. Lett. B 367, 377 (1996); Nucl. Phys. B477, 59 (1996).

[21] J. Papavassiliou and A. Pilaftsis, Phys. Rev. Lett. 80, 2785 (1998).

[22] J. Papavassiliou, E. de Rafael, and N. J. Watson, Nucl. Phys. B503, 79 (1997).

[23] G. 't Hooft and M. Veltman, Nucl. Phys. B153, 365 (1979); G. Passarino and M. Veltman, ibid. B160, 151 (1979).

[24] B. A. Kniehl, Phys. Rep. 240, 211 (1994).
[25] A. Pilaftsis, Phys. Rev. Lett. 77, 4996 (1996); Nucl. Phys. B504, 61 (1997)

[26] J. Papavassiliou, Phys. Rev. D 41, 3179 (1990); 50, 5958 (1994).

[27] B. S. DeWitt, Phys. Rev. 162, 1195 (1967); G. 't Hooft, in Acta Universitatis Wratislvensis No. 38, 12th Winter School of Theoretical Physics in Kapacz, Functional and Probabilistic Methods in Quantum Field Theory, Vol. I (1975), p. 345; B. S. DeWitt, in Quantum Gravity II, edited by C. Isham, R. Penrose, and D. Sciama (Oxford University Press, Oxford, 1981); I. A. Batalin, S. G. Matinian, and G. K. Savvidy, Yad. Fiz. 26, 407 (1977) [Sov. J. Nucl. Phys. 26, 214 (1977)]; L. F. Abbott, Nucl. Phys. B185, 189 (1981); D. M. Capper and A. MacLean, ibid. B203, 413 (1982), and references therein.

[28] A. Denner, S. Dittmaier, and G. Weiglein, Nucl. Phys. B440, 95 (1995).

[29] S. Hashimoto, J. Kodaira, Y. Yasui, and K. Sasaki, Phys. Rev. D 50, 7066 (1994).

[30] A. Pilaftsis, Nucl. Phys. B487, 467 (1997).

[31] See, e.g., J. M. Cornwall, Phys. Rev. D 10, 500 (1974).

[32] N. J. Watson, in Effective Charges in Non-Abelian Gauge Theories, Proceedings of the Ringberg Workshop on Perspectives for electroweak interactions in $e^{+} e^{-}$collisions, Munich, Germany, 1995, edited by B. A. Kniehl, p. 263 [hep-ph/9506276].

[33] N. J. Watson, Nucl. Phys. B494, 388 (1997).

[34] N. J. Watson, Phys. Lett. B 349, 155 (1995).

[35] It is worth emphasizing that in Veltman's pioneering work [36] these issues do not arise, since the toy model he considered was based on $\left(\phi^{3}\right)_{4}$ which is super-renormalizable, i.e., the renormalization group is trivial.

[36] M. Veltman, Physica (Amsterdam) 29, 186 (1963).

[37] A. J. MacFarlane and G. Woo, Nucl. Phys. B77, 91 (1974).

[38] A similar model is treated from a different point of view in Section 8.1 of the book by J. C. Collins, Renormalization (Cambridge University Press, Cambridge, England, 1984).

[39] J. Papavassiliou and K. Philippides, Phys. Rev. D 54, 6957 (1996).

[40] M. Böhm, W. Hollik, and H. Spiesberger, Fortschr. Phys. 34, 687 (1986).

[41] Y.-P. Yao and C.-P. Yuan, Phys. Rev. D 38, 2237 (1988); J. Bagger and C. Schmidt, ibid. 41, 264 (1990); J.-J. He, Y.-P. Kuang, and X. Li, Phys. Rev. Lett. 69, 2619 (1992); Phys. Rev. D 49, 4842 (1994); A. Dobado, J. R. Pelaez, and M. T. Urdiales, ibid. 56, 7133 (1997).

[42] See, e.g., D. Bailin and A. Love, Introduction to Gauge Field Theory (IOP Publishing Ltd., Temple Back, UK, 1993).

[43] Y. J. Feng and C. S. Lam, Phys. Rev. D 56, 6640 (1997), and references therein. 